\title{
Non-Dyadic Interaction: A Literature Review of 15 Years of Human-Robot Interaction Conference Publications
}

\author{
EIKE SCHNEIDERS, Dept. of Computer Science, Aalborg University, Aalborg, Denmark \\ EUNJEONG CHEON, School of Information Studies, Syracuse University, Syracuse, New York \\ JESPER KJELDSKOV, Dept. of Computer Science, Aalborg University, Aalborg, Denmark \\ MATTHIAS REHM, Dept. of Architecture, Design and Media Technology Aalborg University, Aalborg,
}

Denmark

MIKAEL B. SKOV, Dept. of Computer Science, Aalborg University, Aalborg, Denmark

\begin{abstract}
Going beyond dyadic (one-to-one) interaction has been increasingly explored in HRI. Yet we lack a comprehensive view on non-dyadic interaction research in HRI. To map out 15 years of works investigating nondyadic interaction, and thereby identifying the trend of the field and future research areas, we performed a literature review containing all 164 publications (2006-2020) from the HRI conference investigating nondyadic interaction. Our approach is inspired by the $4 \mathrm{C}$ framework, an interaction framework focusing on understanding and categorising different types of interaction between humans and digital artefacts. The $4 \mathrm{C}$ framework consists of eight interaction principles for multi-user/multi-artefact interaction categorised into four broader themes. We modified the $4 \mathrm{C}$ framework to increase applicability and relevance in the context of non-dyadic human-robot interaction. We identify an increasing tendency towards non-dyadic research (36\% in 2020), as well as a focus on simultaneous studies ( $85 \%$ from 2006-2020) over sequential. We also articulate seven interaction principles utilised in non-dyadic HRI and provide specific examples. Last, based on our findings, we discuss several salient points of non-dyadic HRI, the applicability of the modified 4C framework to HRI and potential future topics of interest as well as open-questions for non-dyadic research.
\end{abstract}

CCS Concepts: • Human-centered computing $\rightarrow$ Human computer interaction (HCI); • General and reference $\rightarrow$ Surveys and overviews;

Additional Key Words and Phrases: Non-Dyadic HRI, simultaneous human-robot interaction, literature review

\section{ACM Reference format:}

Eike Schneiders, EunJeong Cheon, Jesper Kjeldskov, Matthias Rehm, and Mikael B. Skov. 2022. Non-Dyadic Interaction: A Literature Review of 15 Years of Human-Robot Interaction Conference Publications. ACM Trans. Hum.-Robot Interact. 11, 2, Article 13 (February 2022), 32 pages.

https://doi.org/10.1145/3488242

\footnotetext{
Authors' addresses: E. Schneiders, J. Kjeldskov, and M. B. Skov, Dept. of Computer Science, Aalborg University, Denmark; emails: \{eike, jesper, dubois\}@cs.aau.dk; E. Cheon, School of Information Studies, Syracuse University, Syracuse, New York; email: echeon@syr.edu; M. Rehm, Dept. of Architecture, Design and Media Technology Aalborg University, Denmark; email: matthias@create.aau.dk.

Permission to make digital or hard copies of all or part of this work for personal or classroom use is granted without fee provided that copies are not made or distributed for profit or commercial advantage and that copies bear this notice and the full citation on the first page. Copyrights for components of this work owned by others than ACM must be honored. Abstracting with credit is permitted. To copy otherwise, or republish, to post on servers or to redistribute to lists, requires prior specific permission and/or a fee. Request permissions from permissions@acm.org. (C) 2022 Association for Computing Machinery.

2573-9522/2022/02-ART13 \$15.00

https://doi.org/10.1145/3488242
} 


\section{INTRODUCTION}

Established in 2006, the ACM/IEEE International Conference on Human-Robot Interaction (HRI conference) is the premiere conference within the field of human-robot interaction (HRI) and has an increasing impact within the field [159]. With the growing maturity of a research field, comprehensive literature reviews are often conducted to map out the field and guide future research (e.g., [7, 39, 148, 160]). For example, Baxter et al. [7] published a literature review of HRI literature from 2013-2015, analysing all 101 publications according to 14 different categories, the main focus being different aspects of the applied methodology. They chose the HRI conference proceedings as the venue for their review, since conference papers give the fastest look into the field, within the domain of peer-reviewed publications. They concluded with six recommendations for future researchers of the interdisciplinary field of HRI. Fischer et al. [39] also identified all HRI conference publications, a final corpus of 27 full papers, covering the topic of emotion expression in robots. They chose the HRI conference, as we do in this article, to gain an overview of current work in emotion expression in HRI. A recent paper by Sebo et al. [161] investigated a corpus of 103 HRI publications from various venues such as HRI conference, RO-MAN, and CSCW. They focus on non-dyadic studies investigating human-robot group configurations, including at least one embodied robot and at least two co-located humans, thereby their focus is on group dynamics in the two configurations one-to-many and many-to-many, while distancing themselves from the many-to-one configuration.

We are currently observing increased attention towards the use of systems including robots interacting with groups of humans as well as mixed groups of robots and other digital artefacts. This tendency shifts the field from a focus on the classical dyadic interaction between one robot and one human (one-to-one interaction) towards more attention on non-dyadic systems involving robots. Oliveira et al. [121] have recently referred to this observation as an ongoing paradigm shift. The same tendency has been observed by Fraune et al. [45], who predict an increase of interaction scenarios involving groups of humans and robots. Further, the need for more focus on non-dyadic studies in HRI has been highlighted on numerous occasions (e.g., [5, 45-47, 71, 95, 121, 122, 189]). Yet, no empirical evidence has been presented documenting this ongoing paradigm shift. Further, the understanding of the characteristics and tendencies of HRI research investigating non-dyadic interaction is still sparse. We believe that the identification of this, through the investigation of current research, is of high value since it can map out current, and past trends in non-dyadic research. Thereby, documenting the paradigm shift and point towards future directions of the research field, ultimately leading to an increased maturity of the still relatively young multidisciplinary field of HRI. We define the non-dyadic interaction in HRI as:

Definition (Non-dyadic Interaction in HRI). An interaction involving at least one physical independent digital artefact-at least one must be an embodied robot-AND at least one human. Either party (artefact OR human) has to be more than one.

The term, physical independent digital artefact, refers to devices independent in physical location from each other. Meaning a robot with a chest-mounted tablet, would not constitute two physically independent artefacts, while the same robot with a detached, movable tablet would. Following this definition, a non-dyadic interaction in HRI can take one of three forms:

(1) One human interacts with multiple digital artefacts, of which at least one is an embodied robot (One-to-Many);

(2) Multiple humans interact with one embodied robot (Many-to-One); 
(3) Multiple humans interact with multiple digital artefacts, of which at least one is an embodied robot (Many-to-Many).

In this article, we conducted a literature review of all full paper publications from the HRI conferences published in 2006-2020. The literature review was inspired by the $4 \mathrm{C}$ framework, a framework to analyse [172] non-dyadic interaction in multi-user/multi-artefact systems. Sørensen et al. [172] developed the the $4 \mathrm{C}$ framework in order to understand different types of interactions in larger systems in which technology users orchestrate multiple digital artefacts and/or humans. The framework takes it's onset in the two axis of configuration (between users and artefacts) as well as the temporal aspect (sequential or simultaneous interaction). Within this $2 \times 2$ grid, four themes (Communality, Continuity, Collaboration, and Complementarity) encapsulate eight interaction principles (Division, Merging, Extension, Remote control, Personalisation, Generalisation, Synchronisation, and Migration).

With foundation in the 4C framework, we investigate HRI conference publications with a particular focus on the following three research questions related to non-dyadic interaction:

$R Q 1$. What current research trends exist in relation to different interaction principles for nondyadic HRI research? And what might this tell us about the distinctive character of nondyadic HRI?

RQ2. How can a multi-user/multi-artefact interaction framework be applied to HRI?

$R Q 3$. What could be future directions for non-dyadic HRI research?

The main contribution of this article is threefold. First, by mapping non-dyadic interaction research on two dimensions, configuration between multi-user/multi-artefact and interaction principle, we highlight current areas of focus as well as gaps in research based on a review of an initial article corpus of 587 full paper publications (2006-2020). Further, we point at trends in relation to dyadic and non-dyadic research over the years, and identify a strong tendency of non-dyadic research in HRI investigating simultaneous interaction. Second, drawing upon the modified 4C framework, we articulate each principle used in non-dyadic interaction, which helps us concretely address, analyse, and discuss the existing and emerging interaction configurations of robots. We further discussed the modified $4 \mathrm{C}$ framework's applicability in the context of HRI. Last, we highlight future directions and open-questions within HRI with a focus on non-dyadic interaction.

The remainder of this article will be structured as follows: In the following section, we describe the applied methodology to identify the relevant publications for this literature review. In Section 3, we present the findings. Following this, Section 4 will discuss the tendencies, the applicability of the $4 \mathrm{C}$ framework to HRI, future research directions for non-dyadic HRI, as well as the limitations of this article.

\section{METHODOLOGY}

In this section, we explore several frameworks which provide a possible lens for the investigation of non-dyadic HRI. We chose the 4C framework for our initial classification and analysis. We further describe the modifications to it to make it fit better into the HRI context. Last, this section presents the process to arrive at the final corpus of articles investigated. The four distinct steps that generated our final corpus are illustrated in Figure 3.

\subsection{Exploring Potential Frameworks for Non-Dyadic Systems}

While we are aware of existing concepts (e.g., the Personal ecology [76], Information ecologies [117]) and frameworks (e.g., Human-Artefact Model [16], Product ecology [43]) for humandigital artefact interaction, many of the existing frameworks focus on one configuration of 
non-dyadic interaction, e.g., the Personal ecology, in which a single human user interacts with digital artefacts (one-to-many). This section will highlight a variety of different lenses that could have been applied.

2.1.1 Product Ecology. Amongst the multitude of frameworks used to understand interaction in systems going beyond dyadic interaction, the product ecology by Forlizzi [43] is one example. Forlizzi proposes the product ecology as a theoretical design framework with multiple purposes. First, the product ecology can be used to understand how product use can evoke social behaviour, and second to help identify appropriate qualitative methods to investigate social product use. As the name implies, the product ecology centres around the individual product, and each product has its own product ecology with different activities, social interaction, and values ascribed to this product. While this would provide a possible theoretical lens for the investigation of non-dyadic HRI, it has some shortcomings concerning the three configurations of non-dyadic interaction investigated in this study. Since the product ecology takes a basis in the individual product, it would be well suited for the investigation of either one-to-one or many-to-one configurations, and therefore be sub-optimal for the approach taken in this article.

2.1.2 Personal Ecology. While the product ecology [43] centres around the product, the personal ecology [76] has the human as the focal point. Jung et al. [76] develop the personal ecology from two studies investigating the artefacts in a person's life and their relations respectively. Through an ecology map study, participant visualised individual artefacts of the ecology and the relations among these. Jung et al. identified three primary layers defining different types of relations between these interactive artefacts. According to Jung et al., these three layers are related to (1) the purpose of the given device (e.g., for "work" or "communication"), (2) the context of use (e.g., "at work" or "carry around, surrounding," and last (3) the subjective meaning people attribute to devices. They conclude that in order to understand the experience and interaction with a given artefact, it does not suffice to investigate this artefact individually. Since the experience with one artefact depends on the integration with other artefacts, the investigation of this requires the consideration of both devices and the relations between these. Thus, the personal ecology would suffice for the investigation of one-to-many interactions, yet another approach would be needed for the remaining two configurations of non-dyadic interaction, namely many-to-one and manyto-many.

2.1.3 Digital Ecologies. By combining the two foci as highlighted by, e.g., Forlizzi [43] and Jung et al. [76], Raptis et al. [144] proposed a standard definition for the term digital ecology. They define three structural levels of relevance for the investigation of digital ecologies. Level one is related to the investigation of a one-to-one nature between human and a digital artefact. Level two describes the interaction in systems with users and all digital artefacts bound by a given activity. Level three removes the activity and considers all digital artefacts in a person's ecology. They define a digital ecology as a set of artefacts, both digital and non-digital, bound by a given activity. Since users can be part of multiple digital ecologies, this definition opens up for the possibility of multi-user interaction with multiple artefacts. Thereby, this view on ecologies satisfies all three non-dyadic configurations of one-to-many, many-to-one, and many-to-many.

As with the product and personal ecology, digital ecology has shortcomings in relation to this study. Raptis et al. [144] present a definition instead of a taxonomy or framework. This makes the "digital ecology" term subpar for this purpose, making it difficult to apply to a given corpus of literature.

While a multitude of frameworks and concepts for the investigation of non-dyadic interaction exists, the existing frameworks typically focus on one configuration, be it on one human with 


\begin{tabular}{|c|c|c|}
\hline & SIMULTANEOUS & SEQUENTIAL \\
\hline $\begin{array}{l}\text { MANY } \\
\text { USERS }\end{array}$ & $\begin{array}{l}\text { Collaboration } \\
\text { - Division } \\
\text { - Merging }\end{array}$ & $\begin{array}{l}\text { Communality } \\
\text { - Personalisation } \\
\text { - Generalisation }\end{array}$ \\
\hline $\begin{array}{l}\text { MANY } \\
\text { ARTEFACTS }\end{array}$ & $\begin{array}{l}\text { Complementarity } \\
\text { - Extension } \\
\text { - Remote Control }\end{array}$ & $\begin{array}{l}\text { Continuity } \\
\text { - Synchronisation } \\
\text { - Migration }\end{array}$ \\
\hline
\end{tabular}

Fig. 1. $2 \times 2$ matrix of the $4 \mathrm{C}$ framework presenting the four themes, eight interaction principles, as well as the two axis simultaneous/sequential and many user/artefact as presented in Sørensen et al. [172].

multiple digital artefacts, or the interaction of multiple humans with one digital artefact. While concepts exist that allow for multi-user/multi-device interaction for all three non-dyadic configurations (e.g., Digital ecology), these are not operationalised, making them hard to apply consistently. In order to investigate all three non-dyadic configurations, we looked for other frameworks with defined terms and principles, which led us to arrive at the $4 \mathrm{C}$ framework. In the following sections, we detail the $4 \mathrm{C}$ framework and the modification we made to adapt it to the context of HRI.

2.1.4 4C Framework. The 4C framework [172] is an interaction framework focusing on understanding and categorising interaction in multi-user/multi-artefact systems. It is a framework for the investigation of different types of interaction categorised by the four themes: Communality, Continuity, Collaboration, and Complementarity. Each theme, as presented in a $2 \times 2$ matrix, has two associated interaction principles resulting in a total of eight interaction principles. The framework further distinguishes between "Many Users" and "Many Artefacts" along the y-axis. Along the $\mathrm{x}$-axis, the framework specifies a temporal aspect, namely the type of interaction being either "Sequential" or "Simultaneous". The original 4C framework, including relations of the themes, interaction principles, user/artefact and temporal axis, can be seen in Figure 1.

"Simultaneous" and "Sequential" interactions typically occur as opposites, yet it is possible to combine both. The same goes for the y-axis, "Many Users" and "Many Artefacts" are, according to the original $4 \mathrm{C}$ framework, minimum requirements. Here we briefly elaborate on the eight interaction principles of the original $4 \mathrm{C}$ framework. Each non-dyadic interaction can include multiple interaction principles.

(1) Collaboration, simultaneous interaction by multiple users.

- Division requires multiple users, just as merging. The distinction here is that every user has a distinct part of the interface. An example would be the interaction between multiple users, each having their own robot as end-point [71].

- Merging relates to the interface used for the interaction. It describes the interaction by multiple users, using the same interface at the same time. An example would be the simultaneous interaction with the same interface provided on a shared touch-table [171].

(2) Complementarity, simultaneous interaction with multiple artefacts.

- Extension describes interaction in which one artefact adds functionality to another artefact. This type of interaction requires a minimum of two physically independent artefacts. An example would be the introduction an interactive wall which extends the robots functionality (by providing it with a virtual counterpart to the physical robot) [151].

- Remote Control describes interaction in which one device controls another device. It differentiates itself from extension by not introducing new functionality, apart from the remote 
controlling functionality. An example would be the teleoperation of robot(s) through e.g., a web interface [50].

(3) Communality, sequential interaction by multiple users.

- Personalisation utilises information about the individual interaction partner to adapt the interaction. An example would be the customisation of interaction, based on user preferences and/or previous user interaction history [80].

- Generalisation relies on no pre-existing knowledge about the individual interaction partner, the interaction with the system will be the same for every user. An example would be the interaction with an ATM, as the interaction does not change depending on the individual user.

(4) Continuity, sequential interaction by multiple artefacts.

- Synchronisation considers interaction that is synchronised between devices. Interaction can be started on a given device and seamlessly continued on a different device. An example would be the seamless transfer of information state between individual robots or artefacts [166].

- Migration contrasts itself from synchronisation by transferring the current system state from one device to another. Instead of maintaining all devices in the interaction in the same state, the current state gets, not duplicated, but migrated. An example would be the migration from implemented code (on e.g., a computer) to a robot to execute it [68]

\subsection{Modifying the 4C Framework for the HRI Context}

The 4C framework provides a well-defined terminology for a specific classification of different interaction techniques along with the two presented axis. Terminological specificity can be beneficial for non-dyadic HRI since it highlights different focus points. While this in itself is useful, after our initial analysis with this framework, we had to modify it in order to increase relevance and decrease ambiguity in the context of HRI. We will describe the specific changes made to the original 4C framework, illustrated in Figure 1, which led to the modified 4C as illustrated in Figure 2.

Type I. Terminological Changes. In terms of terminology, the 4C framework presents four themes and eight interaction principles. In order to adapt this terminology to better suit the HRI context, we made two specific changes.

Since the term "Collaboration" in the context of the $4 \mathrm{C}$ framework strictly refers to interactions involving multiple users (see Figure 1)-which can collaborate with one or several artefacts including robots-this meaning of "Collaboration" is quite different in the context of HRI where the term can refer to dyadic human-robot collaboration. In order to reduce potential connotations associated to the term "Collaboration", we renamed this theme to "Coaction". Thereby, we aimed at keeping the meaning of "working towards a common goal" while countering the problem of the use of the term "Collaboration" which in the original 4C framework is associated with a meaning going against the common understanding of the term in HRI. While it can be argued that most terms, including coaction, might have some connotations to it, we see that the existing use of the term "Coaction" in HRI is less unified and in contradiction to the 4C-frameworks meaning than collaboration within the HRI community. Therefore, it is likely that readers will be less biased towards the term "Coaction".

The second change to the terminology was the change from the term "Communality" to the term "Customisation". Furthermore, as the term "personalisation" and "generalisation" are direct opposites, including both of them is creating redundancy only contributing to a more complex framework, see Section 3. Since personalisation does not require communality, the term 


\begin{tabular}{|c|c|c|c|c|}
\hline \multirow{2}{*}{$\begin{array}{l}\text { CONFIGURATION } \\
\text { Human - Artefact }\end{array}$} & \multicolumn{2}{|c|}{ SIMULTANEOUS } & \multicolumn{2}{|c|}{ SEQUENTIAL } \\
\hline & Complementarity & Coaction & Customisation & Continuity \\
\hline One-to-Many & $\begin{array}{l}\text { - Extension } \\
\text { - Remote Control }\end{array}$ & 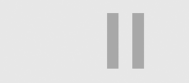 & & $\begin{array}{l}\text { - Synchronisation } \\
\text { - Migration }\end{array}$ \\
\hline Many-to-One & 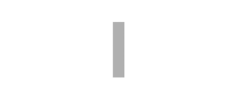 & $\begin{array}{l}\text { - Merging } \\
\text { - Division }\end{array}$ & - Personalisation & \\
\hline Many-to-Many & $\begin{array}{l}\text { - Extension } \\
\text { - Remote Control }\end{array}$ & $\begin{array}{l}\text { - Merging } \\
\text { - Division }\end{array}$ & - Personalisation & $\begin{array}{l}\text { - Synchronisation } \\
\text { - Migration }\end{array}$ \\
\hline
\end{tabular}

Fig. 2. The modified $4 \mathrm{C}$ framework with inspiration. Adapted for the context of non-dyadic human-robot interaction. Fields containing roman numerals (I-IV) are removed since they, by definition, are not possible, see "Type II. Specification of Meaning". This is the final layout of the modified 4C framework that will be used for analysis of the article corpus, which will described in Section 2.3. Results will be presented based on this frameworks format.

"Communality" referring to communal computing [172]), we argue that the term "Communality" seems misleading. We specified the meaning of this by changing the theme name to "Customisation". We thereby attempt to encapsulate the meaning of "personalisation" better.

Type II. Specification of Meaning. While the 4C framework considers different configurations along its y-axis requiring either multiple users or multiple artefacts (or a combination), we wanted to specify each configuration further. Unlike the original 4C framework, we specify the "Many Users" and "Many Artefacts" columns into three configurations (e.g., the number of artefacts which allows for one or more artefacts). In order to illustrate this, we changed the current two rows (Figure 1) to a three-row format (Figure 2). We hope thereby to highlight the configuration possibilities present in the non-dyadic HRI, namely one-to-many, many-to-one or many-to-many. All configurations are to be read as "Human(s)-to-Digital Artefact(s)/Robot(s)".

Since we specify this, the resulting updated 4C framework, as illustrated in Figure 2, has four cells (I - IV) in which which combination of theme and configuration cannot exist.

I For a digital artefact to extend or remote control another, at least two digital artefacts (including robots) are needed, therefore complementarity is not possible in the Many-to-One configuration.

II For an interface to be "Merged" or "Divided" requires at least two users, therefore Coaction can not be used in the One-to-Many configuration.

III For any system to be personalised to the individual, more than one individual has to interact with the system.

IV For a system to be able to migrate or synchronise data from one artefact to another, at least two artefacts need to be present.

Type III. Removing Redundancy. The Communality in the original 4C framework distinguishes between systems that are personalised to each user, e.g., by requiring a login or by adapting the interaction based on individual user performance, and generalisation, which includes systems that do not require any user-specific information. Since "personalisation" and "generalisation" are direct opposites, the 4C framework considers every interaction which does not utilise personalisation as a case of generalisation and vice-versa. Therefore, the inclusion of both principles would create 


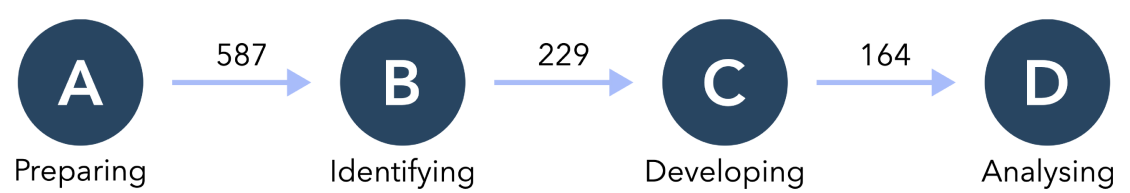

Fig. 3. Methodology used from the preparation (A) of initial corpus, containing 587 full papers, to the analysis (D) of the final corpus, consisting of 164 full papers.

redundancy. Since most publications in the corpus tend to fall into the generalisation, we chose to have only "personalisation" to simplify the framework. As none of the other pairs of interactions principles constitute opposites (e.g., Extension/Remote control, Merging/Division and Synchronisation/Migration), we did not apply this change from two to one principle for additional themes.

\subsection{Corpus Creation}

In this literature review, we investigated all 587 full paper publications at the HRI conferences between 2006-2020, both years included. Inspired by [7, 39], we chose the HRI conference as stateof-the-art conference for peer-reviewed publications within the field of HRI.

(A) Preparing All HRI Publications. We created the initial corpus by downloading all HRI conference full papers from the ACM digital library $(2006-2018,2020)$ as well as the IEEE Xplore digital library (2019). All publications were sorted according to release year in a local repository. This resulted in a total of 587 full papers.

(B) Identifying Non-Dyadic Articles. To ensure that only articles involving the study of non-dyadic HRI are included in the final corpus, all 587 articles were manually scanned. This initial scan, (Figure 3(B)), consisted of a read-through of the title, abstract, keywords, and the introduction. In cases of doubt, the entire article was read with a focus on the methodology and/or experimental procedure. All articles which exclusively studied a dyadic one-to-one interaction between human and robot were removed, leaving only non-dyadic articles in the corpus. As non-dyadic publications, we characterised publications involving multiple digital artefacts, at least one being a physically embodied robot, multiple interacting humans, or both.

(C) Developing the Analysis Template. Of the 229 articles identified during step (B), 60 articles were randomly selected and read to develop an analysis template. The analysis template consisted of 22 points of interest as well as additional information for the unique identification, which was applied to all articles. The analysis template was separated into three distinct categories: "Paper identification", simply used for unique identification of each ; "Article characteristics", this section was split into three sub-categories, identifying information about (1) the interaction(s) studied (e.g., was it sequential or simultaneous, what interaction principle was investigated), (2) the application area(s) studied (e.g., industry, tutoring, domestic); as well as (3) the investigated robot(s) (e.g., models, humanoid, DOF); The last category "Additional Information", contained a summary of the article through a non-dyadic lens in HRI, encountered challenges and opportunities identified in the article. Last, the important findings the article describes were highlighted. The analysis template was, in randomised order, applied to all 229 publications, resulting in the removal of 65 additional publications due to one of the following reasons:

(1) Lack of an embodied robot. This is often the case where the robot is a simulation (e.g., $[56,89,109,199])$ or cases where test subjects participate in a video vignette study (e.g., $[6,111,170])$.

(2) No user involvement (e.g., [82, 103]). 
(3) The contribution of the article was not focused on the interaction, but on topics such as data collection methods, interface design, or framework creation (e.g., [36, 51, 133, 153]).

(4) The article focused on dyadic one-to-one user interaction, which was not visible during step A or B (e.g., [101, 202]).

This resulted in a final corpus of 164 publications investigating non-dyadic HRI for the analysis and extraction of empirical data in step D.

(D) Analysing the Corpus. During step D the 164 articles were quantified into absolute and relative numbers relating to the total amount of publications. Relations between different use contexts, application areas, as well as other metrics were identified to see tendencies within the non-dyadic HRI conferences publications. Further, we applied the terminology as illustrated in Figure 2 (Complementarity, Coaction, Customisation, Continuity). The updated framework characterises different interaction principles with a focus on simultaneous and sequential interaction in multi-user/multi-artefact systems.

For this study, the first author read all publications and classified them in accordance with the $4 \mathrm{C}$ framework and the configuration of multi-user/multi-artefact interaction. When investigating non-dyadic research, we distinguish between three different configurations. These are one-to-many (one human, many digital artefacts of which at least is a robot), many-to-one (many humans, one robot), and many-to-many (many humans, many digital artefacts of which at least is a robot). To ensure the consistent classification of publications into multi-user/multi-artefact configuration, the second author read 25 publications $(15 \%$ of the final corpus) and classified these independently from the first author. We achieved a Cohens' Kappa for the classification into the three different configurations of $\kappa=0.9545$ indicating a high level of inter-rater agreement. Differences were discussed until an agreement could be reached.

We mapped the configuration of interaction (one-to-many, many-to-one, many-to-many) and the seven interaction principles, as presented in Figure 2. For the remainder of this article, all configurations are to be read as: Human(s)-to-Digital Artefact(s)/Robot(s). The findings presented in Section 3 are based on the remaining 164 HRI conference publications.

\section{FINDINGS}

We will start by presenting tendencies within the field. This will be followed by a characterisation of HRI research in relation to simultaneous and sequential research, including the four themes (Complementarity, Coaction, Customisation, and Continuity) and seven principles (Extension, Remote Control, Merging, Division, Personalisation, Synchronisation, and Migration) as presented in the modified $4 \mathrm{C}$ framework, see Figure 2.

\subsection{Tendencies of Non-Dyadic HRI}

The analysed article corpus included 164 HRI papers from the period of 2006-2020. Figure 4 illustrates the tendency in the investigated time period for the HRI conference. The purple line (-) shows absolute numbers and the blue line (-) shows the percentage of non-dyadic publications in relation to all publications. We identified an increase in numbers of HRI papers reporting non-dyadic research over the years. In the early days of the HRI conference, with an exception of 2006/2007, only a few papers focused on non-dyadic research, e.g., only four papers in 2008 (8\%). From 2012, we can see a steady growth in non-dyadic research, resulting in $24(36 \%)$ HRI conference publications investigating non-dyadic interaction in 2020.

When analysing non-dyadic research for HRI, we distinguish between three configurations involving human-robot interaction, as described in Section 2D, (one-to-many, many-to-one, and many-to-many). Out of the 164 publications in our corpus, it is interesting to observe that more 


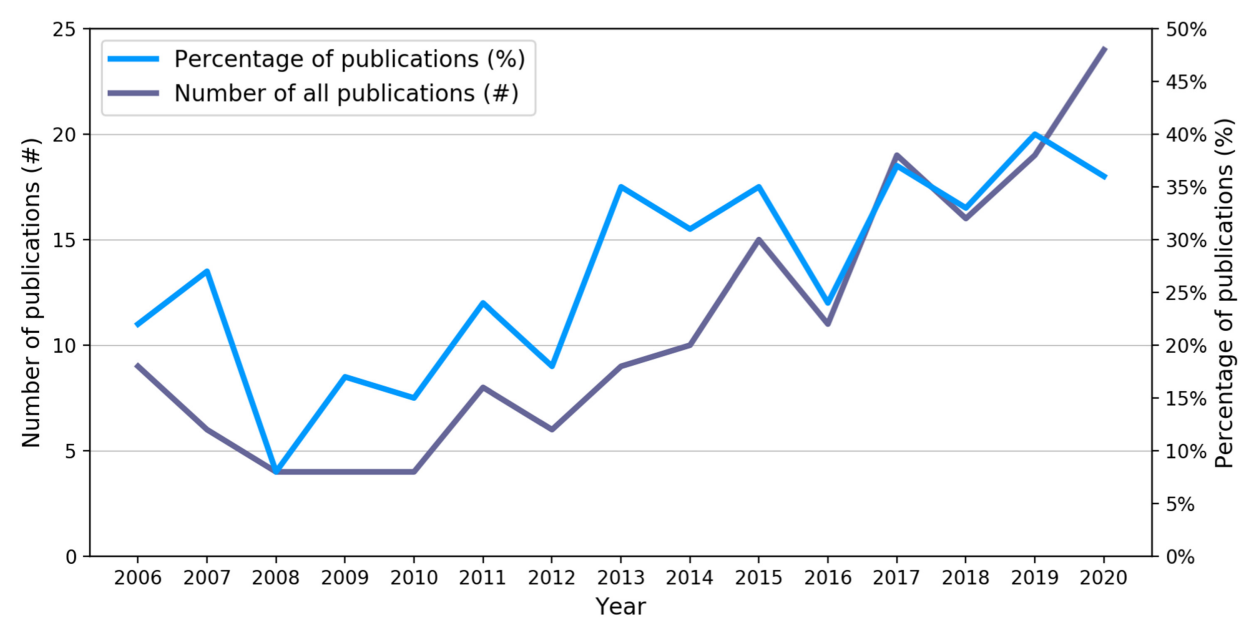

Fig. 4. Visualisation of the number of non-dyadic HRI publications at the HRI conference in absolute numbers (purple line) and in percentage of all publications (blue line).

than half of the papers [85 (51.8\%)] report on one-to-many research. Examples include one human interacting with several robots (e.g., $[3,49,57,127])$ or one human interacting with one robot and other digital artefacts (e.g., [37, 62, 152, 158]). As a recent example of one-to-many research papers, Geiskkovitch et al. [48] illustrated that the trust children direct towards two identical robots significantly differs based on the previous history of statements made by each robot. Many-to-one research was reported in 39 papers $(23.8 \%)$, while many-to-many was the configuration for the remaining 40 papers $(24.4 \%)$. Figure 5 presents the year-over-year trend for the three types of configurations. Interestingly we can see a growth in the later years (from approximately 2016) in the numbers of papers involving one-to-many research. For example, at the HRI conference 2019, 12 $(63 \%)$ of the papers reported one-to-many interaction research (e.g. [25, 162, 198]).

While Figure 4 points at an increased interest and focus on non-dyadic research, Figure 5 shows that this trend, even though increasing for all three configurations, is not evenly distributed. A clear trend, as illustrated by the purple dashed trendline (- --$)$, can be seen for a particular focus on research involving a single user, in a multi-digital artefact environment that includes one to many robots.

\subsection{Simultaneous Interaction}

Simultaneous interaction, also referred to as synchronous interaction [204], can be accomplished in multiple ways, namely through the use of multiple artefacts, the requirement for multiple users or a combination of both. First, multiple digital artefacts, including robots, can be used at the same time by one or many users (e.g., $[63,124,182])$, and second by the simultaneous use of the same digital artefact by multiple users (e.g., $[65,176,184])$. Simultaneous interaction through the lens of the modified $4 \mathrm{C}$ framework makes use of the two themes: complementarity and coaction.

3.2.1 Complementarity. Complementarity is related to the study of interactive systems involving multiple digital artefacts. Since Many-to-One interaction is characterised by having multiple participants but only one digital artefact involved, complementarity is represented as " $N / A$ " in Table 1. Complementarity consists of the two interaction principles extension and remote control [172]. Common for them both is that the artefacts involved in the interaction add to one another. With 117 (71.3\%) articles investigating the theme of complementarity, it is the predominant 


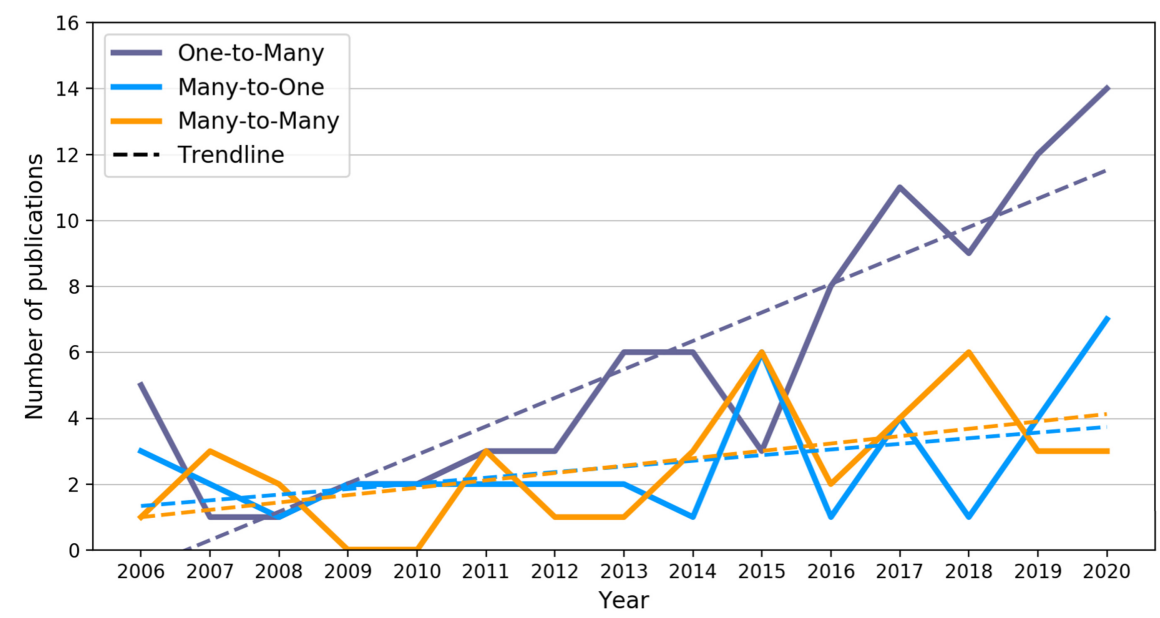

Fig. 5. Illustration of the distribution of the three non-dyadic configurations by year, showing an increase in one-to-many publications compared to the other two configurations. Dashed line is the trendline for each of the three configurations.

theme in the article corpus. Complementarity is typically signified by the interaction of human(s) with multiple robots (e.g., [3, 39, 48, 57]), or human(s) with a robot and other digital artefacts (e.g., $[18,31,67,197])$.

The principle of extension refers to systems in which one artefact adds to another. The principle of extension adds functionality to one artefact through another, see Figure 6(a), thereby distinguishing itself from the closely related principle of remote control. Within the corpus, $79(48.2 \%)$ publications investigated the extension principle. We can observe that extension typically is used in one of two cases. A typical extension investigated is the extension of a robot with an input device such as a touchscreen (e.g., $[18,31,52,66,83,140])$ or laptops (e.g., $[21,104,135,146])$, this extension is observed for 52 of the 79 papers using extension (66\%). An additional case often observed is the extension through the use of multiple robots and tablets, identified for the remaining 27 extension cases $(34 \%)$, in order to increase the functionality of the entire system (e.g., [24, 57, 95, 96, 106]).

An example of the extension principle within HRI is the study by Robert and Breazeal [151]. They developed a room-sized interactive surface (floor and walls) on which children interact with a blended reality robot. The blended reality was caused by the embodied robots' ability to enter a hatch in the wall, seemingly disappearing from the physical space. Simultaneously, it would re-appear as a digital version on the interactive wall. The walls extended the physical embodiment of the robot, which acts as an extension of the robot's physical embodiment with a digital representation.

With $45(27.4 \%)$ publications in the corpus, remote control is the third most represented interaction principle. In contrast to extension, remote control is considering research where one artefact controls another, instead of adding functionality. Within the corpus, the two classical examples for the use of the remote control principle are the two related topics of teleoperation (e.g., [35, 38, 63]) and telepresence (e.g., [136, 174, 188]). Surprisingly, only a minority of the publications that investigate remote control is making use of aerial vehicles in a non-dyadic scenario (e.g., [61, 113, 198]). Remote control is the only interaction principle represented each year related to non-dyadic interaction. With application areas such as search-and-rescue [35], child-robot interaction (CRI) [110], elder-care [8], or the health sector [91], the remote control is a very diverse principle applicable in many different areas. One area of particular research interest within HRI, is the improvement 
Table 1. Final Article Corpus Consisting of $164 \mathrm{HRI}$ conference Publications Investigating Non-Dyadic Interaction from 2006-2020

\begin{tabular}{|c|c|c|c|c|}
\hline & \multicolumn{2}{|c|}{ Simultaneous } & \multicolumn{2}{|c|}{ Sequential } \\
\hline Config. $\quad$ Themes & $\begin{array}{l}\text { Complementarity } \\
(N=117)\end{array}$ & $\begin{array}{l}\text { Coaction } \\
(N=67)\end{array}$ & $\begin{array}{l}\text { Customisation } \\
(N=10)\end{array}$ & $\begin{array}{l}\text { Continuity } \\
(N=5)\end{array}$ \\
\hline $\begin{array}{l}\text { One-to-Many } \\
(N=85)\end{array}$ & $\begin{array}{l}\text { Extension }(N=59): \\
{[2-4,18,20,21,25,28,30,} \\
31,39,45,48,52,53,57,61, \\
62,67,69,70,83,84,92,93, \\
97-99,102,104,106,108, \\
118,119,126-128,135,138, \\
140-142,146,152,155,156, \\
158,162,163,175,186,187, \\
190-192,196-198,205] \\
\text { Remote Control }(N=27): \\
{[1,12,26,33-35,37,38,40,} \\
49,50,61,63,64,78,85,91, \\
94,119,132,137-139,145, \\
178,190,198]\end{array}$ & $N / A$ & N/A & $\begin{array}{l}\text { Synch.: } \\
\text { Migration }(N=4): \\
\begin{array}{ll}68, \quad 134, \quad 173, \\
179]\end{array}\end{array}$ \\
\hline $\begin{array}{l}\text { Many-to-One } \\
(N=39)\end{array}$ & $N / A$ & $\begin{array}{l}\text { Merging }(N=37): \\
{[10,13,17,19,27,29,42,44,} \\
54,55,60,65,74,77,79-81, \\
86-88,107,112,114-116, \\
129,147,165,167,168,176, \\
181,184,195,200,201,203] \\
\text { Division: }\end{array}$ & $\begin{array}{l}\frac{\text { Pers. }(N=7):}{[27,80,105,129,} \\
147,154,176]\end{array}$ & $N / A$ \\
\hline $\begin{array}{l}\text { Many-to-Many } \\
(N=40)\end{array}$ & $\begin{array}{l}\frac{\text { Extension }(N=20):}{[9,22,24,47,59,66,71,95,} \\
96,113,122,124,125,130, \\
151,164,171,177,180,194] \\
\text { Remote Control }(N=18): \\
{[8,9,11,15,23,41,100,} \\
110,113,120,136,150,169, \\
174,182,183,188,206]\end{array}$ & $\begin{array}{l}\text { Merging }(N=18): \\
{[8,22,47,95,100,121,124,} \\
125,151,164,169,171,177, \\
188,189,193,194,206] \\
\text { Division }(N=13): \\
{[9,11,15,24,71,113,120,} \\
122,125,150,174,182,183]\end{array}$ & $\frac{\text { Pers. }(N=3):}{[41,166,180]}$ & $\begin{array}{l}\frac{\text { Synch.: }(N=1):}{[166]} \\
\text { Migration: }\end{array}$ \\
\hline
\end{tabular}

Each paper can be represented by multiple interaction principles, but can only appear in one configuration (Config.). All three configurations are to be read as: "Human(s)-to-Digital Artefact(s)/Robot(s)".

of teleoperation. In order to improve efficiency for remote control in HRI research, leading to the possibility of controlling multiple robots simultaneously, a topic of high attention is the investigation of partial autonomy (e.g., [23, 49, 50, 63, 85, 94, 137, 206]). By combining autonomy and teleoperation, robots only need to prompt the operator whenever an unexpected situation arises, thereby allowing the operator to control multiple robots at a time (e.g., $[49,50,206])$.

An example of a study focusing on research related to improvement the of remote control, through semi-autonomous behaviour, for teleoperation was presented by Glas et al. [50]. They conducted an experiment in a shopping mall in which three previously untrained test subjects, after receiving very limited training time, had to teleoperate four semi-autonomous Robovie-II robots simultaneously in a complex environment, a shopping arcade. They found that the addition of automation capabilities, autonomously switching between the robot in focus, increased the number of interactions with pedestrians as well as the average time between system failures. 


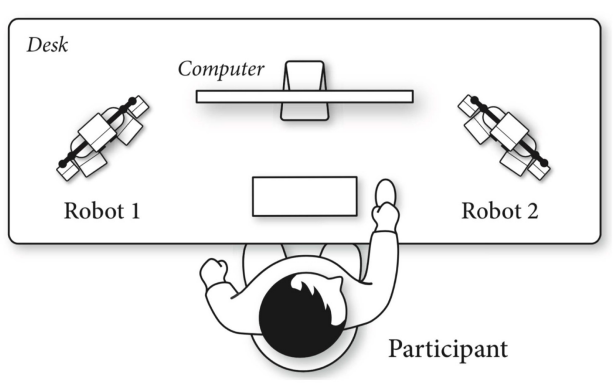

(a)

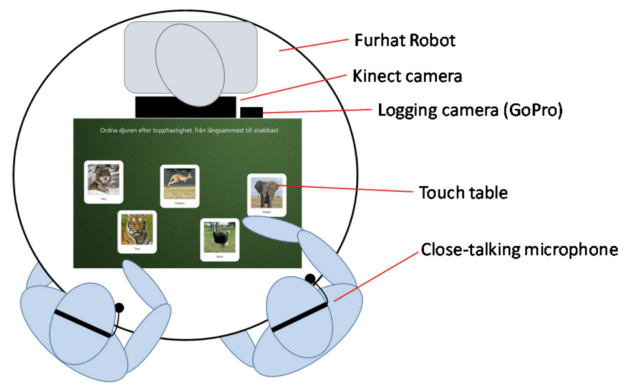

(b)

Fig. 6. (a) Example visualisation of a study utilising the extension principle by Andrist et al. [3] Robot 1 and Robot 2 complement the images presented on the computer through the verbal expression of different degrees of knowledge and rhetorical ability. Thereby, the robots are extending the computers functionality. (b) Skantze et al. [171] make use of coaction, in the illustrated example both human participants interact simultaneously with the furhat robot as well as the touch table, thereby making use of the merging principle.

3.2.2 Coaction. Coaction describes interaction involving multiple users with digital artefacts. It is related to the joint interaction between multiple users with one or more digital artefacts [172]. Due to the requirement for multiple users coaction, as defined by the modified $4 \mathrm{C}$ framework, cannot occur in the one-to-many configuration ("N/A", see Table 1). With a total of $67(40.1 \%)$ publications within the corpus, coaction is the second-largest theme with its two principles division and merging [172]. Coaction has a nearly even distribution between the two configurations, manyto-one and many-to-many, where it is represented with $37(22.6 \%)$ and $30(18.3 \%)$ unique papers, respectively.

When considering the principles under the coaction theme, the principle of merging describes the use of the same parts of the artefact by multiple users, whereas the division principle describes a clear separation of the artefact into distinct sections, each being used by its respective user. For HRI research, a typical example of merged or divided interaction is the use of robots with multiple users, or the use of a tablet with either one collaborative merged user interface or distinct sections for each user.

A multitude of articles document multi-user interaction with the same robot(s) (e.g., [65, 87, $95,124,171,188])$. In total 55 of the $67(82 \%)$ coaction articles have investigated topics related to the merging principle, thereby making merging the second most used interaction principle with a total of $33.5 \%$ of all publications. One of the main characteristics of HRI using merging, is that the device being shared often is limited to the robot(s) (e.g., [60, 80, 168, 203]). Several cases exist in which participants share additional digital artefacts instead of, or beside, the robot such as a connected tablet, screen, or interactive surfaces (e.g., [124, 151, 164]), see Figure 6. We could further observe, that the use of merging is often used in public areas, such as guides in shopping malls (e.g., [13, 80, 81, 167]). Since public space robots have no guarantee about the number of interaction partners at any given time-situations arise in which multiple users collaborate with (or around) the robot, while interacting with the same user interface (such as voice control with the robot), thereby making use of the merging principle in public spaces. Additional examples include merging of interaction with a robot for behaviour change (e.g., $[65,87,184]$ ) as well as teaching (e.g., $[95,124])$.

Using simultaneous multi-user interaction with the same robot device can have profound effects on the group dynamic. An example of merging, is the study by Hoffman et al. [65] where they developed the non-anthropomorphic robot Kip1. Kip1's purpose is to increase people's awareness 
about their behaviour towards others, thereby leading to a potential behaviour change. Kip1 works as a shared robot, visible to both participants of the study, thereby giving them a merged interface with the volume of participants voices as the input modality for the interaction.

To show the viability of merged robot interaction in the context of the classroom, Özgür et al. [124] develop the Cellulo platform, a robotic system of hexagon-shaped interactive robots. To show Cellulos potential for educational purposes, they conduct a study in which groups of three children make use of multiple artefacts: Cellulos, a shared tablet, and a table-sized playground supporting Cellulos self-localisation. In this case, both the playground as well as the tablet are a shared artefact with merged interactivity (although the playground is not digital). The authors show that the merged interface can substitute the teaching experience in a multi-user context.

Even though division is not restricted to cases involving multiple artefacts, all 13 (7.9\%) occurrences of division were in the many-to-many configuration. Just as remote control, division also has a tendency towards studies investigating teleoperation and telepresence (e.g., $[9,15,71,113$, 174]).

One example of a divided interface combined with telepresence, albeit not in the classical sense, through living noise such as the sound of a door opening, is the recent publication by Jeong et al. [71]. They develop the Fribo robot, a robot conveying information represented through living noise produced by each member in a human triad. Each member is via his/her Fribo connected to the other two test participants household, thereby the Fribo provides a clear interface division. By relaying information based on living noise, they try to counter the feeling of loneliness. Jeong et al. [71] illustrate how the division of the interface each user has access to can be used to design for the well-being and improvement in life quality, by countering the feeling of loneliness.

\subsection{Sequential Interaction}

Sequential interaction describes interaction between one or more humans interacting with one or many devices in sequence. For sequential interaction, two themes are defined: customisation and continuity. In this section, we will define these terms as well as illustrate how the interaction principles are used in relation to non-dyadic research in the context of HRI.

3.3.1 Customisation. Customisation describes the interaction with systems of digital artefacts or robots which are customised to the individual interaction partner. Systems in question are typical comprised of digital artefacts or robots, with which multiple users interact, not necessarily at the same time. Since multiple users are involved, this is in direct contradiction with the Oneto-Many configuration as illustrated by " $N / A$ " in Table 1 . Personalisation describes interaction tailored to the individual, therefore requiring data about the individual to optimise the interaction experience (e.g., [154, 180]).

When referring to the term personalisation, represented with $10(6.1 \%)$ occurrences in the corpus, the adjustment of the robot's behaviour or appearance tailored towards the specific individual is described [172], see Figure 7(a). Examples include the use of personalised greeting by the individual's name (e.g., [154]), as well as basing the entire interaction on the user's previous interaction and/or movement history (e.g., [27, 80, 166, 176]). Several positive impacts on contexts such as shopping mall assistance, group-inclusion or elder care of personalisation when interacting with robots have been highlighted.

Personalisation requires interaction with multiple users. While not many examples focusing on this interaction principle exist, a common context is eldercare (e.g. [27, 154]). The recently published study by Cruz-Sandoval et al. [27] investigate the impact of an autonomous robot in a nursing home for dementia patients. They made use of multiple approaches for personalisation, including speaking behaviour fitting for dementia patients or customise the discussion topics 
based on individual patients interests. They found that the personalisation of the robot's behaviour towards the individual had a strong positive impact. They showed enjoyment, higher levels of engagement in the group sessions and in general an increase in mood. Even though the robot led sessions significantly improved typical dementia-related symptoms, they reported that there is no evidence illustrating long-term effects of the personalised interaction.

3.3.2 Continuity. Continuity, represented by 5 (3.0\%) publications in the corpus, describes the interaction involving multiple digital artefacts and is related to an interaction starting on one artefact, and continuing it on a different artefact. Since this involves the transition from one artefact to another, many-to-one is classified as " $N / A$ " (see Table 1 ). This can be achieved by the utilisation of two principles: synchronisation and migration. The synchronisation principle describes the sharing of information from one artefact to another, thereby making sure that the artefacts share the same information base (e.g., [166]). During migration, the state of the users interaction is transferred from one device to another, thereby enabling users to migrate the interaction from one device to another (e.g., $[173,179])$. As illustrated in Table 1, continuity has only been investigated by 5 (3.0\%) and is thereby the least explored theme.

Continuity and the two principles (synchronisation and migration) are only used in a very limited scope in current HRI conference literature (e.g., [166, 173, 179]). Synchronisation investigates systems in which the same knowledge base is shared across multiple devices. While synchronisation is the least used principle, only focused upon in Shiomi et al. [166], it can show a positive impact on the interaction. They illustrated the benefit of synchronising the knowledge space of one robot to multiple other robots to impact the experience of museum visitors. They performed a two-month-long longitudinal study in which over 11,900 RFID tagged users interact with four robots at a museum. The four robots had synchronised knowledge about the user identity, movement pattern, as well as the exhibit-visiting history. Based on this synchronised knowledge among the robots, the robots performed significantly better as a guide.

While not investigated often, migration accounts for the larger part of the continuity-based interaction, $4(2.4 \%)$ publications. The principle of migration describes the process of transferring the state of one's activity from one device to another. The typical context for migration is the programming of robots (e.g. [68, 134, 173]), see Figure 7(b). Stenmark et al. [173], for example, developed an interface for robot programming for non-expert users. The protocol programmed using their interface was migrated to a two-armed robot and executed. They found that even nonexpert users were able to solve the programming tasks, especially when re-using, and combining, move sets programmed by expert users. The vast majority (90\%) of their user base completed the basic level of complexity, whereas even $66 \%$ went beyond this level within the 30 -minute usage.

\section{DISCUSSION}

In this article, we have conducted a systematic literature review of HRI conference papers published during the period 2006-2020 and investigating non-dyadic interaction. Our findings illustrate that the focus on non-dyadic studies has grown over the last 15 years, and now constitutes around $36 \%$ of published papers, compared to, e.g., $8 \%$ in 2008 . Further, we could identify and document a focus in HRI on primarily simultaneous non-dyadic studies. In the following section, we will discuss our three research questions, as presented in Section 1.

\subsection{Simultaneous Interaction as a Dominant Focus of Non-Dyadic HRI}

During our reviews of non-dyadic interaction in HRI, we found aspects which characterise the field of HRI. We found that the studies on non-dyadic interaction in HRI focused nearly exclusively $(85 \%)$ on simultaneous interaction with only a few cases of sequential interaction. 


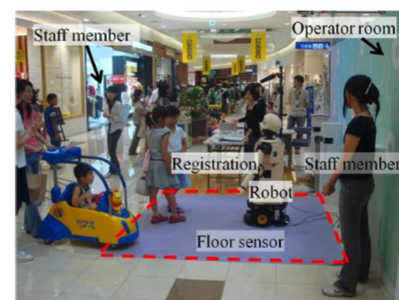

(a)
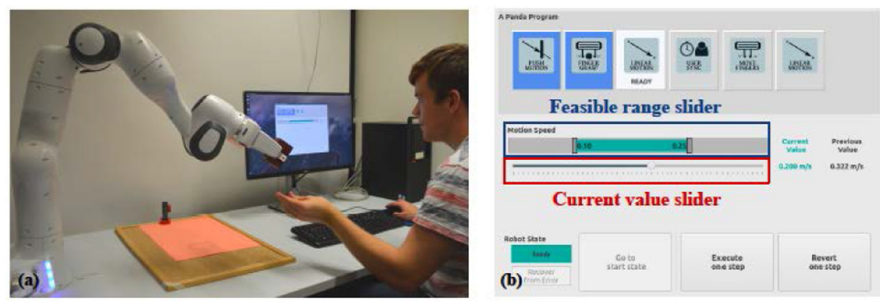

(b)

Fig. 7. (a) Kanda et al. [80] deploy a robotic system in a shopping mall which utilises customisation. Through RFID, the robot builds a history of interaction for each user, and adapts its behaviour through personalisation. (b) Illustration of the continuity theme from Racca et al. [134]. The study uses the principle of migration to adjust the parameters of a robot on one artefact (the PC) and migrate the settings to the robot arm executing the migrated command.

While evidence has been presented [14] showcasing similar tendencies in HCI, non-robotic artefacts have an increased tendency by design to support sequential interaction. Examples of this include typical interactions with non-robotics devices such as smartphones, tablets, and PCs $[32,131,143,157,185])$. Nowadays, it is not uncommon for commercial digital artefacts to be designed with support for sequential interactions, for example, moving sessions between devices [157]. As a corollary to Raptis et al's. [143] findings, the functionality of continuity typically provides increased integration between digital devices and services across multi-devices. For example, Apple's continuity lets users move seamlessly between their iPad, iPhone, Apple Watch, and MacBook through synchronisation and migration. In recent years, simultaneous interactions have also emerged among non-robotic technologies [157]. However, sequential patterns in interaction with non-robotic devices are still frequently used [123], in contrast to interaction with robots. For example, a study by Jokela el al. [73] analyses 123 real-life interactions in non-dyadic systems containing multiple devices, they can demonstrate that $37 \%$ of users (compared to only $15 \%$ in HRI) interact with multiple devices in a sequential manner.

As our findings have shown, extension is the most dominant interaction principle in non-dyadic HRI. Non-robotic technologies (e.g., tablets and laptops) often extended the functionality of robots, observed for 52/79 (66\%) of papers using extension. An example of this is through augmenting of the physical scope of interaction, e.g., through interactive walls [151]. This may imply that robots have sufficient interoperability to integrate them with other digital devices. On the other hand, this implies robots' incompleteness or imperfection in that they typically need the assistance or participation of other devices to function effectively, contrastively reflecting the current discourse of robots representing the most advanced technology.

\subsection{Applicability of the Modified 4C Framework to HRI}

The modified framework helped us unpack a total of 164 non-dyadic interaction cases published at the HRI conference based on seven interaction principles and identify how specific principles are distributed across the HRI studies. In particular, the seven interaction principles allow us to go beyond merely labelling all multi-user and multi-artefact interactions as non-dyadic by focusing on specific mechanisms by which robots can interact with users to achieve their tasks. By articulating each principle of non-dyadic interaction, we could concretely address, analyse, and discuss robots' extant and emergent interaction configurations. 


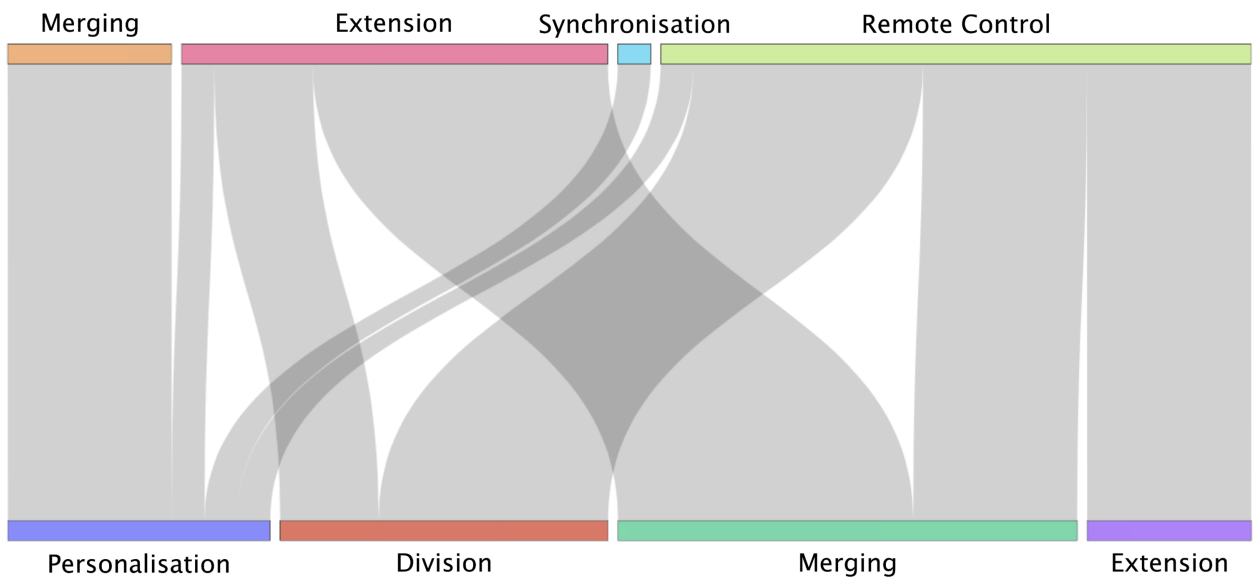

Fig. 8. Sankey diagram illustrating the relations between interaction techniques for all publications in which two interaction principles were present.

Here we reflect our usages of the modified 4C framework in HRI context and share the lessons learned from applying the seven principles to non-dyadic HRI. We hope our reflection will be useful to other researchers using the seven interaction principles in their research. First, we found that it is not common for a single study to utilise more than one non-dyadic interaction principle. Figure 8 illustrates existing relations between publications in the corpus utilising two interaction principles. In the entire corpus, approximately $25 \%$ of papers utilise multiple principles. For example, extension and remote control are the principles frequently combined, since remote control could be seen as one of the means to achieve extension. After reviewing all cases of extension and remote control, we found patterns that distinguished between the two principles. In terms of extension, we looked at whether there was add-on information from other devices, for example, a computers screen displaying a 3D interface or a HoloLens augmenting reality. On the other hand, if there was any control-purpose device (e.g., a joystick or controller) provided for interaction, we considered it remote control. However, we acknowledge some scenarios in which extension and remote control could be interpreted as lying along a continuum, as illustrated by multiple of the papers in the corpus using both principles.

Furthermore, it could be unclear in some cases whether robots should be seen as users rather than artefacts since the modified $4 \mathrm{C}$ framework separates non-dyadic interaction into multi-user and multi-artefact relationships. The separation between users and artefacts might not be so clearcut for robots as for non-robotic technologies because of the robots' strong agency We noted several cases, in which a human participant perceived a robot as another participant in an interaction (e.g., children teaching a robot [18] or a robot generating a co-presence in a workplace [149]). These cases could be more common when robots play a role as collaborators in interaction. Hence, if the modified $4 \mathrm{C}$ framework is to be adapted for HRI, the user matrix should be interpreted broadly to include intelligent agents as users. With that, the non-symmetrical nature [37] between human and robotic agents should also be considered.

While the original 4C framework doesn't differentiate between different configurations, apart from the minimum requirement of either having "Many Users" or "Many Artefacts" as illustrated in Figure 1, the modified 4C framework increases this by specifying three configurations (one-to-many, many-to-one, and many-to-many). This specification added some precision to the framework, yet it could be argued that an even finer granularity is required. One possible change 
could be a further split for the "one-to-many" configuration into the two sub-configurations containing interactions with "multiple robots" and with "one robot and other devices", respectively. Even though we could have made an additional differentiation between "multiple robots" and "one robot and other devices", this would not have changed our resulting framework, as we believe that both types of one-to-many setups can be represented using the same set of interaction principles, as illustrated in Figure 2. When, for instance, looking at the most prominent interaction principle "extension", we see that this occurs both between multiple robots (e.g., [3]) as well as between a robot and additional non-robotic artefacts (e.g., [171]).

\subsection{Future Directions for Non-Dyadic HRI}

As we have presented the overall state of non-dyadic interaction in HRI (see Table 1), there are copious new research opportunities in applying the sequential interaction principles of continuity and customisation to non-dyadic interactions. Here, we first discuss continuity and customisation. Then we propose three open questions for future research directions based on our insights from the overall findings of this study.

A typical case using continuity are service robots (e.g., robots in a museum [166] or reception areas such as a department store, a health clinic, or a hotel [147]), which allow users to interact with the same service agent across multiple robot devices -something which Reig et al. [147] referred to as re-embodiment. For example, Shiomi et al. [166] show that individual visitors interacted with multiple robots at different spots in a museum and that the robots transferred and shared each visitor's input across the robots. As in previous research, the continuity interaction demonstrated that to interact with robots is a promising way to scale up physical spaces. Considering that the continuity takes up the least of the current non-dyadic HRI (3\% of the whole corpus), we hope to see more work in this area.

Regarding personalisation, while only 10 papers (6.1\%) reported on aspects of personalisation in our corpus of papers, there was a strong tendency of reporting positive results of robot personalisation (e.g., [27, 80, 154, 166, 176]). The benefits of personalised systems often derive from a greater willingness to adapt to robots (e.g. [154]) and its effectiveness on peoples behavioural changes (e.g. [166]). Despite the positive impact of personalised robots, ethical issues arise around the possible misuse of personalisation. Since modelling personalisation is based on understanding individual performances and past behaviours, we suggest also considering their potential harms (e.g. manipulating and deceiving users).

Lastly, while we have provided a comprehensive view of the interaction principles for the nondyadic HRI, some aspects remain for further exploration and research. We, here, pose three open questions for future research opportunities:

1. User Experience in Non-Dyadic Interaction. How do different interaction principles affect users' experiences with the interaction?

The modified 4C framework originated from the idea, of (digital) ecologies $[76,117]$ that focuses on experiential aspects of using interconnected technologies. Here, the term ecology is an analogy for describing how people, artefacts and environments are intertwined and how they may influence the experience of interacting with technologies [42]. Drawing upon the idea, of ecology, we propose looking at how different experiences may be created and conveyed by different interaction principles. In our paper we focused on interaction modalities of a non-dyadic interaction by considering one part of ecology: human users and artefacts. One practical way to achieve the experiential aspects would be to additionally consider environments, e.g., social and cultural contexts in which ways of interacting with technologies are affected, as well as the people and activities around the interaction. While this consideration may require new matrix to understand 
the current framework for non-dyadic interaction, this would deepen our understanding of what experiences could be offered through the different interaction principles.

2. Flow of Non-Dyadic Interaction. How may the understanding of a flow of interaction change the current interaction principles?

Another aspect that we suggest is the flow of interaction, i.e., revealing who initiates an interaction and how the interaction proceeds over time. Our analysis mainly focuses on what and who comprises the non-dyadic interaction. However, if we could consider that examining the flow (temporal aspect) of an interaction process, this would allow us to look at the specific roles of humans and devices and further provide a basis for developing the design of interactions. Who (robots or humans) initiates interactions has long been discussed in the domain of human-robot teams [90], leading to the idea, of mixed-initiative interaction [72]. In mixed-initiative interaction, any humans or robots that have the ability to achieve the best results of the tasks assigned can take initiative in an interaction. We believe that different interaction principles might be needed to investigate human- and robot-initiated interactions.

3. Different Types of Tasks in Non-Dyadic HRI. What interaction principles could be effective for different types of tasks?

The final aspect for further inquiry relates to what kind of tasks are used in non-dyadic interaction. As Jokela et al. [73] notes, the type of the task influences the changing of interaction modules (e.g., switching devices or adding another device to their parallel usage of multi devices). Examining what interaction principle might be effective for certain kinds of tasks (e.g., simple or complex execution) could be explored in the future research.

\subsection{Limitations}

This literature review has been conducted only investigating HRI conference publications, thereby limiting the findings to this outlet. It could be argued that alternative outlets could be included to arrive at full coverage of non-dyadic research in HRI. Based on previous literature reviews [7, 39], we choose the HRI conference proceedings as the venue for the review, since it is the most premiere conference within the field of human-robot interaction HRI and has an increasing impact within the field [159].

\section{CONCLUSION}

To achieve a greater understanding of the characteristics and trends of non-dyadic HRI research, we conducted a literature review including all full papers from 2006-2020 investigating non-dyadic literature, with a final corpus of 164 papers. Drawing upon the 4C framework, we made adjustments in order to make it more applicable to the context of HRI resulting in the modified 4C framework where we present its four themes and seven interaction principles along the axis of simultaneous/sequential and specify three interaction configurations (one-to-many, many-to-one, many-to-many). With this modified $4 \mathrm{C}$ framework, we were able to go beyond generally labelling all multi-users/multi-artefact interactions as non-dyadic interactions by focusing on specific mechanisms by which digital artefacts including robots and users employ for interaction and achieve their tasks. As a result, we identified tendencies, such as a strong focus on simultaneous non-dyadic interaction, a growing focus on the one-to-many configuration, as well as areas with little research in HRI (e.g. continuity and customisation in non-dyadic interaction), which is distinct from tendencies in interaction with non-robotics technologies. We further reflected upon the applicability of the modified 4C framework for HRI and brought up two aspects that we want to draw other HRI researchers' attentions when they analyse non-dyadic HRI contexts with this modified 4C 
framework. We finally proposed future directions and open-questions for advancing non-dyadic HRI research.

\section{REFERENCES}

[1] Sigurdur O. Adalgeirsson and Cynthia Breazeal. 2010. Mebot: A robotic platform for socially embodied presence. In Proceedings of the 5th ACM/IEEE International Conference on Human-robot Interaction (Osaka, Japan) (HRI'10). IEEE Press, Piscataway, NJ, 15-22. http://dl.acm.org/citation.cfm?id=1734454.1734467.

[2] Henny Admoni, Bradley Hayes, David Feil-Seifer, Daniel Ullman, and Brian Scassellati. 2013. Are you looking at me? Perception of robot attention is mediated by gaze type and group size. In Proceedings of the 8th ACM/IEEE International Conference on Human-robot Interaction (Tokyo, Japan) (HRI'13). IEEE Press, Piscataway, NJ, 389-396. http://dl.acm.org/citation.cfm?id=2447556.2447685.

[3] Sean Andrist, Erin Spannan, and Bilge Mutlu. 2013. Rhetorical robots: Making robots more effective speakers using linguistic cues of expertise. In Proceedings of the 8th ACM/IEEE International Conference on Human-robot Interaction (Tokyo, Japan) (HRI'13). IEEE Press, Piscataway, NJ, 341-348. http://dl.acm.org/citation.cfm?id=2447556.2447678.

[4] Sean Andrist, Micheline Ziadee, Halim Boukaram, Bilge Mutlu, and Majd Sakr. 2015. Effects of culture on the credibility of robot speech: A comparison between english and arabic. In Proceedings of the 10th Annual ACM/IEEE International Conference on Human-robot Interaction (Portland, Oregon) (HRI'15). ACM, New York, NY, 157-164. https://doi.org/10.1145/2696454.2696464

[5] Thomas Arnold and Matthias Scheutz. 2017. Beyond moral dilemmas: Exploring the ethical landscape in HRI. In Proceedings of the 2017 ACM/IEEE International Conference on Human-robot Interaction (Vienna, Austria) (HRI'17). ACM, New York, NY, 445-452. https://doi.org/10.1145/2909824.3020255

[6] Thomas Arnold and Matthias Scheutz. 2018. Observing robot touch in context: How does touch and attitude affect perceptions of a robot's social qualities? In Proceedings of the 2018 ACM/IEEE International Conference on Humanrobot Interaction (Chicago, IL) (HRI'18). ACM, New York, NY, 352-360. https://doi.org/10.1145/3171221.3171263

[7] Paul Baxter, James Kennedy, Emmanuel Senft, Severin Lemaignan, and Tony Belpaeme. 2016. From characterising three years of HRI to methodology and reporting recommendations. In Proceedings of the 11th ACM/IEEE International Conference on Human Robot Interaction (Christchurch, New Zealand) (HRI'16). IEEE Press, Piscataway, NJ, 391-398. http://dl.acm.org/citation.cfm?id=2906831.2906897.

[8] Jenay M. Beer and Leila Takayama. 2011. Mobile remote presence systems for older adults: Acceptance, benefits, and concerns. In Proceedings of the 6th International Conference on Human-robot Interaction (Lausanne, Switzerland) (HRI'11). ACM, New York, NY, 19-26. https://doi.org/10.1145/1957656.1957665

[9] Chris Bevan and Danaë Stanton Fraser. 2015. Shaking hands and cooperation in tele-present human-robot negotiation. In Proceedings of the 10th Annual ACM/IEEE International Conference on Human-robot Interaction (Portland, Oregon) (HRI'15). ACM, New York, NY, 247-254. https://doi.org/10.1145/2696454.2696490

[10] Serena Booth, James Tompkin, Hanspeter Pfister, Jim Waldo, Krzysztof Gajos, and Radhika Nagpal. 2017. Piggybacking robots: Human-robot overtrust in university dormitory security. In Proceedings of the 2017 ACM/IEEE International Conference on Human-robot Interaction (Vienna, Austria) (HRI'17). ACM, New York, NY, 426-434. https://doi.org/10.1145/2909824.3020211

[11] Paul Bremner and Ute Leonards. 2015. Speech and gesture emphasis effects for robotic and human communicators: A direct comparison. In Proceedings of the 10thAnnual ACM/IEEE International Conference on Human-robot Interaction (Portland, Oregon) (HRI'15). ACM, New York, NY, 255-262. https://doi.org/10.1145/2696454.2696496

[12] C. Brooks and D. Szafir. 2019. Balanced information gathering and goal-oriented actions in shared autonomy. In Proceedings of the 2019 14th ACM/IEEE International Conference on Human-robot Interaction (HRI). 85-94. https://doi. org/10.1109/HRI.2019.8673192

[13] Drazen Brscić, Hiroyuki Kidokoro, Yoshitaka Suehiro, and Takayuki Kanda. 2015. Escaping from children's abuse of social robots. In Proceedings of the 10th Annual ACM/IEEE International Conference on Human-robot Interaction (Portland, Oregon) (HRI'15). ACM, New York, NY, 59-66. https://doi.org/10.1145/2696454.2696468

[14] Frederik Brudy, Christian Holz, Roman Rädle, Chi-Jui Wu, Steven Houben, Clemens Nylandsted Klokmose, and Nicolai Marquardt. 2019. Cross-device taxonomy: Survey, opportunities and challenges of interactions spanning across multiple devices. In Proceedings of the 2019 CHI Conference on Human Factors in Computing Systems (Glasgow, Scotland UK) (CHI'19). ACM, New York, NY, Article 562, 28 pages. https://doi.org/10.1145/3290605.3300792

[15] Jenny Burke and Robin Murphy. 2007. RSVP: An investigation of remote shared visual presence as common ground for human-robot teams. In Proceedings of the ACM/IEEE International Conference on Human-robot Interaction (Arlington, Virginia) (HRI'07). ACM, New York, NY, 161-168. https://doi.org/10.1145/1228716.1228738

[16] Susanne Bødker and ClemensNylandsted Klokmose. 2013. The human-artifact model: An activity theoretical approach to artifact ecologies. Hum.-comput. Interact. 26, 4 (Dec. 2013), 315-371. 
[17] Sonja Caraian, Nathan Kirchner, and Peter Colborne-Veel. 2015. Moderating a robot's ability to influence people through its level of sociocontextual interactivity. In Proceedings of the 10th Annual ACM/IEEE International Conference on Human-robot Interaction (Portland, Oregon) (HRI'15). ACM, New York, NY, 149-156. https://doi.org/10.1145/ 2696454.2696489

[18] Shruti Chandra, Raul Paradeda, Hang Yin, Pierre Dillenbourg, Rui Prada, and Ana Paiva. 2018. Do children perceive whether a robotic peer is learning or not? In Proceedings of the 2018 ACM/IEEE International Conference on Humanrobot Interaction (Chicago, IL) (HRI'18). ACM, New York, NY, 41-49. https://doi.org/10.1145/3171221.3171274

[19] Wan-Ling Chang and Selma Šabanović. 2015. Interaction expands function: Social shaping of the therapeutic robot PARO in a nursing home. In Proceedings of the 10th Annual ACM/IEEE International Conference on Human-robot Interaction (Portland, Oregon) (HRI'15). ACM, New York, NY, 343-350. https://doi.org/10.1145/2696454.2696472

[20] Huili Chen, Hae Won Park, Xiajie Zhang, and Cynthia Breazeal. 2020. Impact of interaction context on the student affect-learning relationship in child-robot interaction. In Proceedings of the 2020 ACM/IEEE International Conference on Human-robot Interaction (Cambridge, United Kingdom) (HRI'20). Association for Computing Machinery, New York, NY, 389-397. https://doi.org/10.1145/3319502.3374822

[21] Vijay Chidambaram, Yueh-Hsuan Chiang, and Bilge Mutlu. 2012. Designing persuasive robots: How robots might persuade people using vocal and nonverbal cues. In Proceedings of the 7th Annual ACM/IEEE International Conference on Human-robot Interaction (Boston, Massachusetts) (HRI'12). ACM, New York, NY, 293-300. https://doi.org/10.1145/ 2157689.2157798

[22] Joe Connolly, Viola Mocz, Nicole Salomons, Joseph Valdez, Nathan Tsoi, Brian Scassellati, and Marynel Vázquez. 2020. Prompting prosocial human interventions in response to robot mistreatment. In Proceedings of the 2020 ACM/IEEE International Conference on Human-robot Interaction (Cambridge, United Kingdom) (HRI'20). Association for Computing Machinery, New York, NY, 211-220. https://doi.org/10.1145/3319502.3374781

[23] Andrew Correa, Matthew R. Walter, Luke Fletcher, Jim Glass, Seth Teller, and Randall Davis. 2010. Multimodal interaction with an autonomous forklift. In Proceedings of the 5th ACM/IEEE International Conference on Human-robot Interaction (Osaka, Japan) (HRI'10). IEEE Press, Piscataway, NJ, 243-250. http://dl.acm.org/citation.cfm?id=1734454.1734550.

[24] Filipa Correia, Samuel Mascarenhas, Rui Prada, Francisco S. Melo, and Ana Paiva. 2018. Group-based emotions in teams of humans and robots. In Proceedings of the 2018 ACM/IEEE International Conference on Human-robot Interaction (Chicago, IL, USA) (HRI’18). ACM, New York, NY, 261-269. https://doi.org/10.1145/3171221.3171252

[25] F. Correia, S. F. Mascarenhas, S. Gomes, P. Arriaga, I. Leite, R. Prada, F. S. Melo, and A. Paiva. 2019. Exploring prosociality in human-robot teams. In Proceedings of the 2019 14th ACM/IEEE International Conference on Humanrobot Interaction (HRI'19). ACM, New York, NY, 143-151. https://doi.org/10.1109/HRI.2019.8673299

[26] Christopher Crick, Sarah Osentoski, Graylin Jay, and Odest Chadwicke Jenkins. 2011. Human and robot perception in large-scale learning from demonstration. In Proceedings of the 6th International Conference on Human-robot Interaction (Lausanne, Switzerland) (HRI'11). ACM, New York, NY, 339-346. https://doi.org/10.1145/1957656.1957788

[27] Dagoberto Cruz-Sandoval, Arturo Morales-Tellez, Eduardo Benitez Sandoval, and Jesus Favela. 2020. A social robot as therapy facilitator in interventions to deal with dementia-related behavioral symptoms. In Proceedings of the 2020 ACM/IEEE International Conference on Human-robot Interaction (Cambridge, United Kingdom) (HRI'20). Association for Computing Machinery, New York, NY, 161-169. https://doi.org/10.1145/3319502.3374840

[28] Daniel P. Davison, Frances M. Wijnen, Vicky Charisi, Jan van der Meij, Vanessa Evers, and Dennis Reidsma. 2020. Working with a social robot in school: A long-term real-world unsupervised deployment. In Proceedings of the 2020 ACM/IEEE International Conference on Human-robot Interaction (Cambridge, United Kingdom) (HRI'20). Association for Computing Machinery, New York, NY, 63-72. https://doi.org/10.1145/3319502.3374803

[29] Maartje de Graaf, Somaya Ben Allouch, and Jan van Dijk. 2017. Why do they refuse to use my robot? Reasons for nonuse derived from a long-term home study. In Proceedings of the 2017 ACM/IEEE International Conference on Humanrobot Interaction (Vienna, Austria) (HRI'17). ACM, New York, NY, 224-233. https://doi.org/10.1145/2909824.3020236

[30] Jan de Wit, Arold Brandse, Emiel Krahmer, and Paul Vogt. 2020. Varied human-like gestures for social robots: Investigating the effects on children's engagement and language learning. In Proceedings of the 2020 ACM/IEEE International Conference on Human-robot Interaction (Cambridge, United Kingdom) (HRI'20). Association for Computing Machinery, New York, NY, 359-367. https://doi.org/10.1145/3319502.3374815

[31] Jan de Wit, Thorsten Schodde, Bram Willemsen, Kirsten Bergmann, Mirjam de Haas, Stefan Kopp, Emiel Krahmer, and Paul Vogt. 2018. The effect of a robot's gestures and adaptive tutoring on children's acquisition of second language vocabularies. In Proceedings of the 2018 ACM/IEEE International Conference on Human-robot Interaction (Chicago, IL) (HRI'18). ACM, New York, NY, 50-58. https://doi.org/10.1145/3171221.3171277

[32] David Dearman and Jeffery S. Pierce. 2008. It's on my other computer! Computing with multiple devices. In Proceedings of the SIGCHI Conference on Human Factors in Computing Systems. 767-776.

[33] Joseph DelPreto and Daniela Rus. 2020. Plug-and-play gesture control using muscle and motion sensors. In Proceedings of the 2020 ACM/IEEE International Conference on Human-robot Interaction (Cambridge, United Kingdom) (HRI'20). Association for Computing Machinery, New York, NY, 439-448. https://doi.org/10.1145/3319502.3374823 
[34] Munjal Desai, Poornima Kaniarasu, Mikhail Medvedev, Aaron Steinfeld, and Holly Yanco. 2013. Impact of robot failures and feedback on real-time trust. In Proceedings of the 8th ACM/IEEE International Conference on Human-robot Interaction (Tokyo, Japan) (HRI'13). IEEE Press, Piscataway, NJ, 251-258. http://dl.acm.org/citation.cfm?id=2447556. 2447663.

[35] Munjal Desai, Mikhail Medvedev, Marynel Vázquez, Sean McSheehy, Sofia Gadea-Omelchenko, Christian Bruggeman, Aaron Steinfeld, and Holly Yanco. 2012. Effects of changing reliability on trust of robot systems. In Proceedings of the 7thh Annual ACM/IEEE International Conference on Human-robot Interaction (Boston, Massachusetts) (HRI'12). ACM, New York, NY, 73-80. https://doi.org/10.1145/2157689.2157702

[36] Jill L. Drury, Jean Scholtz, and David Kieras. 2007. Adapting GOMS to model human-robot interaction. In Proceedings of the ACM/IEEE International Conference on Human-robot Interaction (Arlington, Virginia) (HRI'07). ACM, New York, NY, 41-48. https://doi.org/10.1145/1228716.1228723

[37] Jill L. Drury, Holly A. Yanco, Whitney Howell, Brian Minten, and Jennifer Casper. 2006. Changing shape: Improving situation awareness for a polymorphic robot. In Proceedings of the 1st ACM SIGCHI/SIGART Conference on Humanrobot Interaction (Salt Lake City, Utah) (HRI'06). ACM, New York, NY, 72-79. https://doi.org/10.1145/1121241.1121256

[38] François Ferland, François Pomerleau, Chon Tam Le Dinh, and François Michaud. 2009. Egocentric and exocentric teleoperation interface using real-time, 3D video projection. In Proceedings of the 4th ACM/IEEE International Conference on Human Robot Interaction (La Jolla, California) (HRI'09). ACM, New York, NY, 37-44. https://doi.org/10.1145/ 1514095.1514105

[39] K. Fischer, M. Jung, L. C. Jensen, and M. V. aus der Wieschen. 2019. Emotion expression in HRI - when and why. In Proceedings of the 2019 14th ACM/IEEE International Conference on Human-robot Interaction (HRI'19). ACM, New York, NY. 29-38. https://doi.org/10.1109/HRI.2019.8673078

[40] Kerstin Fischer, Franziska Kirstein, Lars Christian Jensen, Norbert Krüger, Kamil Kukliński, Maria Vanessa aus der Wieschen, and Thiusius Rajeeth Savarimuthu. 2016. A comparison of types of robot control for programming by demonstration. In Proceedings of the 11th ACM/IEEE International Conference on Human Robot Interaction (Christchurch, New Zealand) (HRI'16). IEEE Press, Piscataway, NJ, 213-220. http://dl.acm.org/citation.cfm?id= 2906831.2906868.

[41] Naomi T. Fitter, Luke Rush, Elizabeth Cha, Thomas Groechel, Maja J. Matarić, and Leila Takayama. 2020. Closeness is key over long distances: Effects of interpersonal closeness on telepresence experience. In Proceedings of the 2020 ACM/IEEE International Conference on Human-robot Interaction (Cambridge, United Kingdom) (HRI'20). Association for Computing Machinery, New York, NY, 499-507. https://doi.org/10.1145/3319502.3374785

[42] Jodi Forlizzi. 2007. How robotic products become social products: An ethnographic study of cleaning in the home. In Proceedings of the ACM/IEEE International Conference on Human-robot Interaction (Arlington, Virginia) (HRI'07) ACM, New York, NY, 129-136. https://doi.org/10.1145/1228716.1228734

[43] Jodi Forlizzi. 2008. The product ecology: Understanding social product use and supporting design culture. International fournal of Design; Vol 2, No 1 (2008) (2008). http://www.ijdesign.org/index.php/IJDesign/article/view/220.

[44] Jodi Forlizzi and Carl DiSalvo. 2006. Service robots in the domestic environment: A study of the Roomba vacuum in the home. In Proceedings of the 1st ACM SIGCHI/SIGART Conference on Human-robot Interaction (Salt Lake City, Utah) (HRI'06). ACM, New York, NY, 258-265. https://doi.org/10.1145/1121241.1121286

[45] Marlena R. Fraune, Yusaku Nishiwaki, Selma Sabanović, Eliot R. Smith, and Michio Okada. 2017. Threatening flocks and mindful snowflakes: How group entitativity affects perceptions of robots. In Proceedings of the 2017 ACM/IEEE International Conference on Human-robot Interaction (Vienna, Austria) (HRI'17). ACM, New York, NY, 205-213. https: //doi.org/10.1145/2909824.3020248

[46] Marlena R. Fraune, Steven Sherrin, Selma Sabanović, and Eliot R. Smith. 2015. Rabble of robots effects: Number and type of robots modulates attitudes, emotions, and stereotypes. In Proceedings of the 10th Annual ACM/IEEE International Conference on Human-robot Interaction (Portland, Oregon) (HRI'15). ACM, New York, NY, 109-116. https: //doi.org/10.1145/2696454.2696483

[47] M. R. Fraune, S. Sherrin, S. Šabanović, and E. R. Smith. 2019. Is human-robot interaction more competitive between groups than between individuals? In Proceedings of the 2019 14th ACM/IEEE International Conference on Human-robot Interaction (HRI'19). ACM, New York, NY, 104-113. https://doi.org/10.1109/HRI.2019.8673241

[48] D. Y. Geiskkovitch, R. Thiessen, J. E. Young, and M. R. Glenwright. 2019. What? That's not a chair!: How robot informational errors affect children's trust towards robots. In Proceedings of the 2019 14th ACM/IEEE International Conference on Human-robot Interaction (HRI'19). ACM, New York, NY, 48-56. https://doi.org/10.1109/HRI.2019.8673024

[49] Dylan F. Glas, Takayuki Kanda, Hiroshi Ishiguro, and Norihiro Hagita. 2008. Simultaneous teleoperation of multiple social robots. In Proceedings of the 3rd ACM/IEEE International Conference on Human Robot Interaction (Amsterdam, The Netherlands) (HRI'08). ACM, New York, NY, 311-318. https://doi.org/10.1145/1349822.1349863

[50] Dylan F. Glas, Takayuki Kanda, Hiroshi Ishiguro, and Norihiro Hagita. 2009. Field trial for simultaneous teleoperation of mobile social robots. In Proceedings of the 4th ACM/IEEE International Conference on Human Robot Interaction (La Jolla, California) (HRI'09). ACM, New York, NY, 149-156. https://doi.org/10.1145/1514095.1514123 
[51] Michael A. Goodrich, Timothy W. McLain, Jeffrey D. Anderson, Jisang Sun, and Jacob W. Crandall. 2007. Managing autonomy in robot teams: Observations from four experiments. In Proceedings of the ACM/IEEE International Conference on Human-robot Interaction (Arlington, Virginia) (HRI'07). ACM, New York, NY, 25-32. https: //doi.org/10.1145/1228716.1228721

[52] Goren Gordon, Cynthia Breazeal, and Susan Engel. 2015. Can children catch curiosity from a social robot? In Proceedings of the 10th Annual ACM/IEEE International Conference on Human-robot Interaction (Portland, Oregon) (HRI'15). ACM, New York, NY, 91-98. https://doi.org/10.1145/2696454.2696469

[53] Arzu Guneysu Ozgur, Maximilian Jonas Wessel, Wafa Johal, Kshitij Sharma, Ayberk Özgür, Philippe Vuadens, Francesco Mondada, Friedhelm Christoph Hummel, and Pierre Dillenbourg. 2018. Iterative design of an upper limb rehabilitation game with tangible robots. In Proceedings of the 2018 ACM/IEEE International Conference on Humanrobot Interaction (Chicago, IL) (HRI'18). ACM, New York, NY, 241-250. https://doi.org/10.1145/3171221.3171262

[54] Omer Gvirsman, Yaacov Koren, Tal Norman, and Goren Gordon. 2020. Patricc: A platform for triadic interaction with changeable characters. In Proceedings of the 2020 ACM/IEEE International Conference on Human-robot Interaction (Cambridge, United Kingdom) (HRI'20). Association for Computing Machinery, New York, NY, 399-407. https://doi. org $/ 10.1145 / 3319502.3374792$

[55] Marc Hanheide, Denise Hebesberger, and Tomáš Krajník. 2017. The when, where, and how: An adaptive robotic info-terminal for care home residents. In Proceedings of the 2017 ACM/IEEE International Conference on Human-robot Interaction (Vienna, Austria) (HRI'17). ACM, New York, NY, 341-349. https://doi.org/10.1145/2909824.3020228

[56] Benjamin Hardin and Michael A. Goodrich. 2009. On using mixed-initiative control: A perspective for managing large-scale robotic teams. In Proceedings of the 4th ACM/IEEE International Conference on Human Robot Interaction (La Jolla, California) (HRI'09). ACM, New York, NY, 165-172. https://doi.org/10.1145/1514095.1514126

[57] Markus Häring, Dieta Kuchenbrandt, and Elisabeth André. 2014. Would you like to play with me? How robots' group membership and task features influence human-robot interaction. In Proceedings of the 2014 ACM/IEEE International Conference on Human-robot Interaction (Bielefeld, Germany) (HRI'14). ACM, New York, NY, 9-16. https://doi.org/10. $1145 / 2559636.2559673$

[58] Mojgan Hashemian, Ana Paiva, Samuel Mascarenhas, Pedro A. Santos, and Rui Prada. 2019. Social power in humanrobot interaction: Towards more persuasive robots. In Proceedings of the 18th International Conference on Autonomous Agents and MultiAgent Systems. 2015-2017.

[59] Kotaro Hayashi, Daisuke Sakamoto, Takayuki Kanda, Masahiro Shiomi, Satoshi Koizumi, Hiroshi Ishiguro, Tsukasa Ogasawara, and Norihiro Hagita. 2007. Humanoid robots as a passive-social medium: A field experiment at a train station. In Proceedings of the ACM/IEEE International Conference on Human-robot Interaction (Arlington, Virginia) (HRI'07). ACM, New York, NY, 137-144. https://doi.org/10.1145/1228716.1228735

[60] Denise Hebesberger, Christian Dondrup, Tobias Koertner, Christoph Gisinger, and Juergen Pripfl. 2016. Lessons learned from the deployment of a long-term autonomous robot as companion in physical therapy for older adults with dementia: A mixed methods study. In Proceedings of the 11th ACM/IEEE International Conference on Human Robot Interaction (Christchurch, New Zealand) (HRI'16). IEEE Press, Piscataway, NJ, 27-34. http://dl.acm.org/citation. cfm?id=2906831.2906838

[61] Hooman Hedayati, Michael Walker, and Daniel Szafir. 2018. Improving collocated robot teleoperation with augmented reality. In Proceedings of the 2018 ACM/IEEE International Conference on Human-robot Interaction (Chicago, IL) (HRI'18). ACM, New York, NY, 78-86. https://doi.org/10.1145/3171221.3171251

[62] Jacqueline Hemminghaus and Stefan Kopp. 2017. Towards adaptive social behavior generation for assistive robots using reinforcement learning. In Proceedings of the 2017 ACM/IEEE International Conference on Human-robot Interaction (Vienna, Austria) (HRI'17). ACM, New York, NY, A, 332-340. https://doi.org/10.1145/2909824.3020217

[63] Laura V. Herlant, Rachel M. Holladay, and Siddhartha S. Srinivasa. 2016. Assistive teleoperation of robot arms via automatic time-optimal mode switching. In Proceedings of the 11th ACM/IEEE International Conference on Human Robot Interaction (Christchurch, New Zealand) (HRI'16). IEEE Press, Piscataway, NJ, 35-42. http://dl.acm.org/citation. cfm?id=2906831.2906839.

[64] Susan G. Hill and Barry Bodt. 2007. A field experiment of autonomous mobility: Operator workload for one and two robots. In Proceedings of the ACM/IEEE International Conference on Human-robot Interaction (Arlington, Virginia) (HRI'07). ACM, New York, NY, 169-176. https://doi.org/10.1145/1228716.1228739

[65] Guy Hoffman, Oren Zuckerman, Gilad Hirschberger, Michal Luria, and Tal Shani Sherman. 2015. Design and evaluation of a peripheral robotic conversation companion. In Proceedings of the 10th Annual ACM/IEEE International Conference on Human-robot Interaction (Portland, Oregon) (HRI'15). ACM, New York, NY, 3-10. https://doi.org/10. $1145 / 2696454.2696495$

[66] Deanna Hood, Séverin Lemaignan, and Pierre Dillenbourg. 2015. When children teach a robot to write: An autonomous teachable humanoid which uses simulated handwriting. In Proceedings of the 10th Annual ACM/IEEE International Conference on Human-robot Interaction (Portland, Oregon) (HRI'15). ACM, New York, NY, 83-90. https: //doi.org/10.1145/2696454.2696479 
[67] Chien-Ming Huang and Bilge Mutlu. 2014. Learning-based modeling of multimodal behaviors for humanlike robots. In Proceedings of the 2014 ACM/IEEE International Conference on Human-robot Interaction (Bielefeld, Germany) (HRI'14). ACM, New York, NY, A, 57-64. https://doi.org/10.1145/2559636.2559668

[68] Justin Huang and Maya Cakmak. 2017. Code3: A system for end-to-end programming of mobile manipulator robots for novices and experts. In Proceedings of the 2017 ACM/IEEE International Conference on Human-robot Interaction (Vienna, Austria) (HRI'17). ACM, New York, NY, 453-462. https://doi.org/10.1145/2909824.3020215

[69] Justin Huang, Tessa Lau, and Maya Cakmak. 2016. Design and evaluation of a rapid programming system for service robots. In Proceedings of the 11th ACM/IEEE International Conference on Human Robot Interaction (Christchurch, New Zealand) (HRI'16). IEEE Press, Piscataway, NJ, 295-302. http://dl.acm.org/citation.cfm?id=2906831.2906883.

[70] Alexis D. Jacq, Séverin Lemaignan, Fernando Garcia, Pierre Dillenbourg, and Ana Paiva. 2016. Building successful long child-robot interactions in a learning context. In Proceedings of the 11th ACM/IEEE International Conference on Human Robot Interaction (Christchurch, New Zealand) (HRI'16). IEEE Press, Piscataway, NJ, 239-246. http://dl.acm. org/citation.cfm?id=2906831.2906874.

[71] Kwangmin Jeong, Jihyun Sung, Hae-Sung Lee, Aram Kim, Hyemi Kim, Chanmi Park, Yuin Jeong, JeeHang Lee, and Jinwoo Kim. 2018. FRIBO: A social networking robot for increasing social connectedness through sharing daily home activities from living noise data. In Proceedings of the 2018 ACM/IEEE International Conference on Human-robot Interaction (Chicago, IL) (HRI'18). ACM, New York, NY, 114-122. https://doi.org/10.1145/3171221.3171254

[72] Shu Jiang and Ronald C. Arkin. 2015. Mixed-initiative human-robot interaction: Definition, taxonomy, and survey. In Proceedings of the 2015 IEEE International Conference on Systems, Man, and Cybernetics. IEEE, 954-961.

[73] Tero Jokela, Jarno Ojala, and Thomas Olsson. 2015. A diary study on combining multiple information devices in everyday activities and tasks. In Proceedings of the 33rd Annual ACM Conference on Human Factors in Computing Systems (Seoul, Republic of Korea) (CHI'15). Association for Computing Machinery, New York, NY, 3903-3912. https: //doi.org/10.1145/2702123.2702211

[74] S. Joshi and S. Šabanović. 2019. Robots for inter-generational interactions: Implications for nonfamilial community settings. In Proceedings of the 2019 14th ACM/IEEE International Conference on Human-robot Interaction (HRI'14). 478486. https://doi.org/10.1109/HRI.2019.8673167

[75] Wendy Ju. 2016. Power in human robot interactions. In Robophilosophy/Transor. 13-14.

[76] Heekyoung Jung, Erik Stolterman, Will Ryan, Tonya Thompson, and Marty Siegel. 2008. Toward a framework for ecologies of artifacts: How are digital artifacts interconnected within a personal life? In Proceedings of the 5th Nordic Conference on Human-computer Interaction: Building Bridges. 201-210.

[77] Malte F. Jung, Nikolas Martelaro, and Pamela J. Hinds. 2015. Using robots to moderate team conflict: The case of repairing violations. In Proceedings of the 10th Annual ACM/IEEE International Conference on Human-robot Interaction (Portland, Oregon) (HRI'15). ACM, New York, NY, 229-236. https://doi.org/10.1145/2696454.2696460

[78] M. Waleed Kadous, Raymond Ka-Man Sheh, and Claude Sammut. 2006. Effective user interface design for rescue robotics. In Proceedings of the 1st ACM SIGCHI/SIGART Conference on Human-robot Interaction (Salt Lake City, Utah) (HRI’06). ACM, New York, NY, 250-257. https://doi.org/10.1145/1121241.1121285

[79] Takayuki Kanda, Michihiro Shimada, and Satoshi Koizumi. 2012. Children learning with a social robot. In Proceedings of the 7th Annual ACM/IEEE International Conference on Human-robot Interaction (Boston, Massachusetts) (HRI'12) ACM, New York, NY, 351-358. https://doi.org/10.1145/2157689.2157809

[80] Takayuki Kanda, Masahiro Shiomi, Zenta Miyashita, Hiroshi Ishiguro, and Norihiro Hagita. 2009. An affective guide robot in a shopping mall. In Proceedings of the 4th ACM/IEEE International Conference on Human Robot Interaction (La Jolla, California) (HRI'09). ACM, New York, NY, 173-180. https://doi.org/10.1145/1514095.1514127

[81] Yusuke Kato, Takayuki Kanda, and Hiroshi Ishiguro. 2015. May I help you? Design of human-like polite approaching behavior. In Proceedings of the 10th Annual ACM/IEEE International Conference on Human-robot Interaction (Portland, Oregon) (HRI'15). ACM, New York, NY, 35-42. https://doi.org/10.1145/2696454.2696463

[82] Charles C. Kemp, Cressel D. Anderson, Hai Nguyen, Alexander J. Trevor, and Zhe Xu. 2008. A point-and-click interface for the real world: Laser designation of objects for mobile manipulation. In Proceedings of the 3rd ACM/IEEE International Conference on Human Robot Interaction (Amsterdam, The Netherlands) (HRI'08). ACM, New York, NY, 241-248. https://doi.org/10.1145/1349822.1349854

[83] James Kennedy, Paul Baxter, and Tony Belpaeme. 2015. The robot who tried too hard: Social behaviour of a robot tutor can negatively affect child learning. In Proceedings of the 10th Annual ACM/IEEE International Conference on Humanrobot Interaction (Portland, Oregon) (HRI'15). ACM, New York, NY, 67-74. https://doi.org/10.1145/2696454.2696457

[84] James Kennedy, Paul Baxter, Emmanuel Senft, and Tony Belpaeme. 2016. Social robot tutoring for child second language learning. In Proceedings of the 11th ACM/IEEE International Conference on Human Robot Interaction (Christchurch, New Zealand) (HRI'16). IEEE Press, Piscataway, NJ, 231-238. http://dl.acm.org/citation.cfm?id= 2906831.2906873. 
[85] David Kent, Carl Saldanha, and Sonia Chernova. 2017. A comparison of remote robot teleoperation interfaces for general object manipulation. In Proceedings of the 2017 ACM/IEEE International Conference on Human-robot Interaction (Vienna, Austria) (HRI'17). ACM, New York, NY, 371-379. https://doi.org/10.1145/2909824.3020249

[86] Hiroyuki Kidokoro, Takayuki Kanda, Dražen Brščic, and Masahiro Shiomi. 2013. Will I bother here? A robot anticipating its influence on pedestrian walking comfort. In Proceedings of the 8th ACM/IEEE International Conference on Human-robot Interaction (Tokyo, Japan) (HRI'13). IEEE Press, Piscataway, NJ, 259-266. http://dl.acm.org/citation. cfm?id=2447556.2447664.

[87] Yuki Kinoshita, Masanori Yokoyama, Shigeo Yoshida, Takayoshi Mochizuki, Tomohiro Yamada, Takuji Narumi, Tomohiro Tanikawa, and Michitaka Hirose. 2017. Transgazer: Improving impression by switching direct and averted gaze using optical illusion. In Proceedings of the 2017 ACM/IEEE International Conference on Human-robot Interaction (Vienna, Austria) (HRI'17). ACM, New York, NY, 53-62. https://doi.org/10.1145/2909824.3020235

[88] Nathan Kirchner, Alen Alempijevic, and Gamini Dissanayake. 2011. Nonverbal robot-group interaction using an imitated gaze cue. In Proceedings of the 6th International Conference on Human-robot Interaction (Lausanne, Switzerland) (HRI'11). ACM, New York, NY, 497-504. https://doi.org/10.1145/1957656.1957824

[89] Andreas Kolling, Steven Nunnally, and Michael Lewis. 2012. Towards human control of robot swarms. In Proceedings of the 7th Annual ACM/IEEE International Conference on Human-robot Interaction (Boston, Massachusetts) (HRI'12). ACM, New York, NY, 89-96. https://doi.org/10.1145/2157689.2157704

[90] David Kortenkamp, R. Peter Bonasso, Dan Ryan, and Debbie Schreckenghost. 1997. Traded control with autonomous robots as mixed initiative interaction. In Proceedings of the AAAI Symposium on Mixed Initiative Interaction. 89-94.

[91] Kory Kraft and William D. Smart. 2016. Seeing is comforting: Effects of teleoperator visibility in robot-mediated health care. In Proceedings of the 11th ACM/IEEE International Conference on Human Robot Interaction (Christchurch, New Zealand) (HRI'16). IEEE Press, Piscataway, NJ, 11-18. http://dl.acm.org/citation.cfm?id=2906831.2906836.

[92] A. Kshirsagar, B. Dreyfuss, G. Ishai, O. Heffetz, and G. Hoffman. 2019. Monetary-incentive competition between humans and robots: Experimental results. In Proceedings of the 2019 14th ACM/IEEE International Conference on Humanrobot Interaction (HRI'19). ACM, New York, NY, 95-103. https://doi.org/10.1109/HRI.2019.8673201

[93] Alyssa Kubota, Emma I. C. Peterson, Vaishali Rajendren, Hadas Kress-Gazit, and Laurel D. Riek. 2020. JESSIE: Synthesizing social robot behaviors for personalized neurorehabilitation and beyond. In Proceedings of the 2020 ACM/IEEE International Conference on Human-robot Interaction (Cambridge, United Kingdom) (HRI'20). Association for Computing Machinery, New York, NY, 121-130. https://doi.org/10.1145/3319502.3374836

[94] Adam Eric Leeper, Kaijen Hsiao, Matei Ciocarlie, Leila Takayama, and David Gossow. 2012. Strategies for humanin-the-loop robotic grasping. In Proceedings of the 7th Annual ACM/IEEE International Conference on Human-robot Interaction (Boston, Massachusetts) (HRI'12). ACM, New York, NY, 1-8. https://doi.org/10.1145/2157689.2157691

[95] Iolanda Leite, Marissa McCoy, Monika Lohani, Daniel Ullman, Nicole Salomons, Charlene Stokes, Susan Rivers, and Brian Scassellati. 2015. Emotional storytelling in the classroom: Individual versus group interaction between children and robots. In Proceedings of the 10th Annual ACM/IEEE International Conference on Human-robot Interaction (Portland, Oregon) (HRI'15). ACM, New York, NY, 75-82. https://doi.org/10.1145/2696454.2696481

[96] Iolanda Leite, Marissa McCoy, Daniel Ullman, Nicole Salomons, and Brian Scassellati. 2015. Comparing models of disengagement in individual and group interactions. In Proceedings of the 10th Annual ACM/IEEE International Conference on Human-robot Interaction (Portland, Oregon) (HRI'15). ACM, New York, NY, 99-105. https://doi.org/10.1145/ 2696454.2696466

[97] Séverin Lemaignan, Fernando Garcia, Alexis Jacq, and Pierre Dillenbourg. 2016. From real-time attention assessment to "with-me-ness" in human-robot interaction. In Proeedings of the 11th ACM/IEEE International Conference on Human-robot Interaction (Christchurch, New Zealand) (HRI'16). IEEE Press, Piscataway, NJ, 157-164. http: //dl.acm.org/citation.cfm?id=2906831.2906860.

[98] Dan Leyzberg, Eleanor Avrunin, Jenny Liu, and Brian Scassellati. 2011. Robots that express emotion elicit better human teaching. In Proceedings of the 6th International Conference on Human-robot Interaction (Lausanne, Switzerland) (HRI'11). ACM, New York, NY, 347-354. https://doi.org/10.1145/1957656.1957789

[99] Daniel Leyzberg, Samuel Spaulding, and Brian Scassellati. 2014. Personalizing robot tutors to individuals' learning differences. In Proceedings of the 2014 ACM/IEEE International Conference on Human-robot Interaction (Bielefeld, Germany) (HRI'14). ACM, New York, NY, 423-430. https://doi.org/10.1145/2559636.2559671

[100] Chaoran Liu, Carlos T. Ishi, and Hiroshi Ishiguro. 2015. Bringing the scene back to the tele-operator: Auditory scene manipulation for tele-presence systems. In Proceedings of the 10th Annual ACM/IEEE International Conference on Human-robot Interaction (Portland, Oregon) (HRI'15). ACM, New York, NY, 279-286. https://doi.org/10.1145/2696454. 2696494

[101] Gale M. Lucas, Jill Boberg, David Traum, Ron Artstein, Jonathan Gratch, Alesia Gainer, Emmanuel Johnson, Anton Leuski, and Mikio Nakano. 2018. Getting to know each other: The role of social dialogue in recovery from errors in social robots. In Proceedings of the 2018 ACM/IEEE International Conference on Human-robot Interaction (Chicago, IL) (HRI'18). ACM, New York, NY, 344-351. https://doi.org/10.1145/3171221.3171258 
[102] Jason P. Luck, Patricia L. McDermott, Laurel Allender, and Deborah C. Russell. 2006. An investigation of real world control of robotic assets under communication latency. In Proceedings of the 1st ACM SIGCHI/SIGART Conference on Human-robot Interaction (Salt Lake City, Utah) (HRI'06). ACM, New York, NY, 202-209. https://doi.org/10.1145/ 1121241.1121277

[103] Raafat Ahmed Abdel El-Azim Mahmoud, Atsushi Ueno, and Shoji Tatsumi. 2011. An assistive tele-operated anthropomorphic robot hand: Osaka city university hand II. In Proceedings of the 6th International Conference on Human-robot Interaction (Lausanne, Switzerland) (HRI'11). ACM, New York, NY, 85-92. https://doi.org/10.1145/1957656.1957677

[104] Maxim Makatchev, Reid Simmons, Majd Sakr, and Micheline Ziadee. 2013. Expressing ethnicity through behaviors of a robot character. In Proceedings of the 8th ACM/IEEE International Conference on Human-robot Interaction (Tokyo, Japan) (HRI'13). IEEE Press, Piscataway, NJ, 357-364. http://dl.acm.org/citation.cfm?id=2447556.2447681.

[105] Eric Martinson, Wallace Lawson, and Greg Trafton. 2013. Identifying people with soft-biometrics at fleet week. In Proceedings of the 8th ACM/IEEE International Conference on Human-robot Interaction (Tokyo, Japan) (HRI'13). IEEE Press, Piscataway, NJ, 49-56. http://dl.acm.org/citation.cfm?id=2447556.2447565.

[106] Takahiro Matsumoto, Mitsuhiro Goto, Ryo Ishii, Tomoki Watanabe, Tomohiro Yamada, and Michita Imai. 2018. Where should robots talk? Spatial arrangement study from a participant workload perspective. In Proceedings of the 2018 ACM/IEEE International Conference on Human-robot Interaction (Chicago, IL) (HRI'18). ACM, New York, NY, 270-278. https://doi.org/10.1145/3171221.3171265

[107] C. Mavrogiannis, A. M. Hutchinson, J. MacDonald, P. Alves-Oliveira, and R. A. Knepper. 2019. Effects of distinct robot navigation strategies on human behavior in a crowded environment. In 2019 14th ACM/IEEE International Conference on Human-robot Interaction (HRI'19). 421-430. https://doi.org/10.1109/HRI.2019.8673115

[108] Carlo Mazzola, Alexander Mois Aroyo, Francesco Rea, and Alessandra Sciutti. 2020. Interacting with a social robot affects visual perception of space. In Proceedings of the 2020 ACM/IEEE International Conference on Human-robot Interaction (Cambridge, United Kingdom) (HRI'20). Association for Computing Machinery, New York, NY, 549-557. https://doi.org/10.1145/3319502.3374819

[109] Samuel J. McDonald, Mark B. Colton, C. Kristopher Alder, and Michael A. Goodrich. 2017. Haptic shape-based management of robot teams in cordon and patrol. In Proceedings of the 2017 ACM/IEEE International Conference on Humanrobot Interaction (Vienna, Austria) (HRI'17). ACM, New York, NY, 380-388. https://doi.org/10.1145/2909824.3020243

[110] Marek P. Michalowski, Selma Sabanovic, and Hideki Kozima. 2007. A dancing robot for rhythmic social interaction. In Proceedings of the ACM/IEEE International Conference on Human-robot Interaction (Arlington, Virginia) (HRI'07). ACM, New York, NY, 89-96. https://doi.org/10.1145/1228716.1228729

[111] Maryam Moosaei, Sumit K. Das, Dan O. Popa, and Laurel D. Riek. 2017. Using facially expressive robots to calibrate clinical pain perception. In Proceedings of the 2017 ACM/IEEE International Conference on Human-robot Interaction (Vienna, Austria) (HRI'17). ACM, New York, NY, 32-41. https://doi.org/10.1145/2909824.3020216

[112] Lilia Moshkina, Susan Trickett, and J. Gregory Trafton. 2014. Social engagement in public places: A tale of one robot. In Proceedings of the 2014 ACM/IEEE International Conference on Human-robot Interaction (Bielefeld, Germany) (HRI'14). ACM, New York, NY, 382-389. https://doi.org/10.1145/2559636.2559678

[113] Robin R. Murphy, Kevin S. Pratt, and Jennifer L. Burke. 2008. Crew roles and operational protocols for rotary-wing micro-uavs in close urban environments. In Proceedings of the $3 r d$ ACM/IEEE International Conference on Human Robot Interaction (Amsterdam, The Netherlands) (HRI'08). ACM, New York, NY, 73-80. https://doi.org/10.1145/1349822. 1349833

[114] Bilge Mutlu and Jodi Forlizzi. 2008. Robots in organizations: The role of workflow, social, and environmental factors in human-robot interaction. In Proceedings of the 3rd ACM/IEEE International Conference on Human Robot Interaction (Amsterdam, The Netherlands) (HRI'08). ACM, New York, NY, 287-294. https://doi.org/10.1145/1349822.1349860

[115] Bilge Mutlu, Toshiyuki Shiwa, Takayuki Kanda, Hiroshi Ishiguro, and Norihiro Hagita. 2009. Footing in humanrobot conversations: How robots might shape participant roles using gaze cues. In Proceedings of the 4th ACM/IEEE International Conference on Human Robot Interaction (La Jolla, California) (HRI'09). ACM, New York, NY, 61-68. https: //doi.org/10.1145/1514095.1514109

[116] Shogo Nabe, Takayuki Kanda, Kazuo Hiraki, Hiroshi Ishiguro, Kiyoshi Kogure, and Norihiro Hagita. 2006. Analysis of human behavior to a communication robot in an open field. In Proceedings of the 1st ACM SIGCHI/SIGART Conference on Human-robot Interaction (Salt Lake City, Utah) (HRI'06). ACM, New York, NY, 234-241. https://doi.org/10.1145/ 1121241.1121282

[117] Bonnie A. Nardi and Vicki L. O’Day. 1999. Information Ecologies: Using Technology with Heart. MIT Press.

[118] Manisha Natarajan and Matthew Gombolay. 2020. Effects of anthropomorphism and accountability on trust in human-robot interaction. In Proceedings of the 2020 ACM/IEEE International Conference on Human-robot Interaction (Cambridge, United Kingdom) (HRI'20). Association for Computing Machinery, New York, NY, 33-42. https: //doi.org/10.1145/3319502.3374839 
[119] Curtis W. Nielsen and Michael A. Goodrich. 2006. Comparing the usefulness of video and map information in navigation tasks. In Proceedings of the 1st ACM SIGCHI/SIGART Conference on Human-robot Interaction (Salt Lake City, Utah, USA) (HRI'06). ACM, New York, NY, USA, 95-101. https://doi.org/10.1145/1121241.1121259

[120] Domen Novak, Aniket Nagle, and Robert Riener. 2014. Can two-player games increase motivation in rehabilitation robotics? In Proceedings of the 2014 ACM/IEEE International Conference on Human-robot Interaction (Bielefeld, Germany) (HRI'14). ACM, New York, NY, 447-454. https://doi.org/10.1145/2559636.2559658

[121] Raquel Oliveira, Patrícia Arriaga, Patrícia Alves-Oliveira, Filipa Correia, Sofia Petisca, and Ana Paiva. 2018. Friends or foes? Socioemotional support and gaze behaviors in mixed groups of humans and robots. In Proceedings of the 2018 ACM/IEEE International Conference on Human-robot Interaction (Chicago, IL) (HRI'18). ACM, New York, NY, 279-288. https://doi.org/10.1145/3171221.3171272

[122] R. Oliveira, P. Arriaga, F. Correia, and A. Paiva. 2019. The stereotype content model applied to human-robot interactions in groups. In Proceedings of the 2019 14th ACM/IEEE International Conference on Human-robot Interaction (HRI). 123-132. https://doi.org/10.1109/HRI.2019.8673171

[123] Antti Oulasvirta and Lauri Sumari. 2007. Mobile kits and laptop trays: Managing multiple devices in mobile information work. In Proceedings of the SIGCHI Conference on Human Factors in Computing Systems. 1127-1136.

[124] Ayberk Özgür, Wafa Johal, Francesco Mondada, and Pierre Dillenbourg. 2017. Windfield: Learning wind meteorology with handheld haptic robots. In Proceedings of the 2017 ACM/IEEE International Conference on Human-robot Interaction (Vienna, Austria) (HRI'17). ACM, New York, NY, 156-165. https://doi.org/10.1145/2909824.3020231

[125] Ayberk Òzgür, Séverin Lemaignan, Wafa Johal, Maria Beltran, Manon Briod, Léa Pereyre, Francesco Mondada, and Pierre Dillenbourg. 2017. Cellulo: Versatile handheld robots for education. In Proceedings of the 2017 ACM/IEEE International Conference on Human-robot Interaction (Vienna, Austria) (HRI'17). ACM, New York, NY, 119-127. https://doi.org/10.1145/2909824.3020247

[126] Maike Paetzel, Giulia Perugia, and Ginevra Castellano. 2020. The persistence of first impressions: The effect of repeated interactions on the perception of a social robot. In Proceedings of the 2020 ACM/IEEE International Conference on Human-robot Interaction (Cambridge, United Kingdom) (HRI'20). Association for Computing Machinery, New York, NY, 73-82. https://doi.org/10.1145/3319502.3374786

[127] Hae Won Park, Mirko Gelsomini, Jin Joo Lee, and Cynthia Breazeal. 2017. Telling stories to robots: The effect of backchanneling on a child's storytelling. In Proceedings of the 2017 ACM/IEEE International Conference on Humanrobot Interaction (Vienna, Austria) (HRI'17). ACM, New York, NY, 100-108. https://doi.org/10.1145/2909824.3020245

[128] Hae Won Park, Rinat Rosenberg-Kima, Maor Rosenberg, Goren Gordon, and Cynthia Breazeal. 2017. Growing growth mindset with a social robot peer. In Proceedings of the 2017 ACM/IEEE International Conference on Human-robot Interaction (Vienna, Austria) (HRI'17). ACM, New York, NY, 137-145. https://doi.org/10.1145/2909824.3020213

[129] Hannah R. M. Pelikan, Mathias Broth, and Leelo Keevallik. 2020. "Are you sad, Cozmo?” How humans make sense of a home robot's emotion displays. In Proceedings of the 2020 ACM/IEEE International Conference on Human-robot Interaction (Cambridge, United Kingdom) (HRI'20). Association for Computing Machinery, New York, NY, 461-470. https://doi.org/10.1145/3319502.3374814

[130] F. Petric and Z. Kovačić. 2019. Hierarchical POMDP framework for a robot-assisted ASD diagnostic protocol. In Proceedings of the 2019 14th ACM/IEEE International Conference on Human-robot Interaction (HRI'19). ACM, New York, 286-293. https://doi.org/10.1109/HRI.2019.8673295

[131] Jeffrey S. Pierce and Jeffrey Nichols. 2008. An infrastructure for extending applications' user experiences across multiple personal devices. In Proceedings of the 21st Annual ACM Symposium on User Interface Software and Technology. ACM, New York, 101-110.

[132] Pragathi Praveena, Daniel Rakita, Bilge Mutlu, and Michael Gleicher. 2020. Supporting perception of weight through motion-induced sensory conflicts in robot teleoperation. In Proceedings of the 2020 ACM/IEEE International Conference on Human-robot Interaction (Cambridge, United Kingdom) (HRI'20). Association for Computing Machinery, New York, NY, 509-517. https://doi.org/10.1145/3319502.3374841

[133] Erin Pudenz, Geb Thomas, Justin Glasgow, Peter Coppin, David Wettergreen, and Nathalie Cabrol. 2006. Searching for a quantitative proxy for rover science effectiveness. In Proceedings of the 1st ACM SIGCHI/SIGART Conference on Human-robot Interaction (Salt Lake City, Utah) (HRI'06). ACM, New York, NY, 18-25. https://doi.org/10.1145/1121241. 1121247

[134] Mattia Racca, Ville Kyrki, and Maya Cakmak. 2020. Interactive tuning of robot program parameters via expected divergence maximization. In Proceedings of the 2020 ACM/IEEE International Conference on Human-robot Interaction (Cambridge, United Kingdom) (HRI'20). Association for Computing Machinery, New York, NY, 629-638. https://doi. org/10.1145/3319502.3374784

[135] M. Racca, A. Oulasvirta, and V. Kyrki. 2019. Teacher-aware active robot learning. In Proceedings of the 2019 14th ACM/IEEE International Conference on Human-robot Interaction (HRI). ACM, New York, 335-343. https://doi.org/10. 1109/HRI.2019.8673300 
[136] Irene Rae, Leila Takayama, and Bilge Mutlu. 2013. The influence of height in robot-mediated communication. In Proceedings of the 8th ACM/IEEE International Conference on Human-robot Interaction (Tokyo, Japan) (HRI'13). IEEE Press, Piscataway, NJ, 1-8. http://dl.acm.org/citation.cfm?id=2447556.2447558.

[137] Daniel Rakita, Bilge Mutlu, and Michael Gleicher. 2017. A motion retargeting method for effective mimicry-based teleoperation of robot arms. In Proceedings of the 2017 ACM/IEEE International Conference on Human-robot Interaction (Vienna, Austria) (HRI'17). ACM, New York, NY, 361-370. https://doi.org/10.1145/2909824.3020254

[138] Daniel Rakita, Bilge Mutlu, and Michael Gleicher. 2018. An autonomous dynamic camera method for effective remote teleoperation. In Proceedings of the 2018 ACM/IEEE International Conference on Human-robot Interaction (Chicago, IL) (HRI'18). ACM, New York, NY, 325-333. https://doi.org/10.1145/3171221.3171279

[139] Daniel Rakita, Bilge Mutlu, and Michael Gleicher. 2020. Effects of onset latency and robot speed delays on mimicrycontrol teleoperation. In Proceedings of the 2020 ACM/IEEE International Conference on Human-robot Interaction (Cambridge, United Kingdom) (HRI'20). Association for Computing Machinery, New York, NY, 519-527. https: //doi.org/10.1145/3319502.3374838

[140] Aditi Ramachandran, Chien-Ming Huang, Edward Gartland, and Brian Scassellati. 2018. Thinking aloud with a tutoring robot to enhance learning. In Proceedings of the 2018 ACM/IEEE International Conference on Human-robot Interaction (Chicago, IL, USA) (HRI'18). ACM, New York, NY, USA, 59-68. https://doi.org/10.1145/3171221.3171250

[141] Aditi Ramachandran, Chien-Ming Huang, and Brian Scassellati. 2017. Give me a break!: Personalized timing strategies to promote learning in robot-child tutoring. In Proceedings of the 2017 ACM/IEEE International Conference on Human-robot Interaction (Vienna, Austria) (HRI'17). ACM, New York, NY, 146-155. https://doi.org/10.1145/2909824. 3020209

[142] Aditi Ramachandran, Alexandru Litoiu, and Brian Scassellati. 2016. Shaping productive help-seeking behavior during robot-child tutoring interactions. In Proceedings of the 11th ACM/IEEE International Conference on Human Robot Interaction (Christchurch, New Zealand) (HRI'16). IEEE Press, Piscataway, NJ, 247-254. http://dl.acm.org/citation. cfm?id=2906831.2906875.

[143] Dimitrios Raptis, Jesper Kjeldskov, and Mikael B. Skov. 2016. Continuity in multi-device interaction: An online study. In Proceedings of the 9th Nordic Conference on Human-computer Interaction (Gothenburg, Sweden) (NordiCHI'16). ACM, New York, NY, Article 29, 10 pages. https://doi.org/10.1145/2971485.2971533

[144] Dimitrios Raptis, Jesper Kjeldskov, Mikael B. Skov, and Jeni Paay. 2014. What is a digital ecology? Theoretical foundations and a unified definition. Australian fournal of Intelligent Information Processing Systems 13, 4 (2014), 5.

[145] D. J. Rea and J. E. Young. 2019. Backseat teleoperator: Affective feedback with on-screen agents to influence teleoperation. In Proceedings of the 2019 14th ACM/IEEE International Conference on Human-robot Interaction (HRI'19). 19-28. https://doi.org/10.1109/HRI.2019.8673014

[146] Natalia Reich-Stiebert and Friederike Eyssel. 2017. (Ir)Relevance of Gender? On the Influence of Gender Stereotypes on Learning with a Robot. In Proceedings of the 2017 ACM/IEEE International Conference on Human-robot Interaction (Vienna, Austria) (HRI'17). ACM, New York, NY, 166-176. https://doi.org/10.1145/2909824.3020242

[147] Samantha Reig, Michal Luria, Janet Z. Wang, Danielle Oltman, Elizabeth Jeanne Carter, Aaron Steinfeld, Jodi Forlizzi, and John Zimmerman. 2020. Not some random agent: Multi-person interaction with a personalizing service robot. In Proceedings of the 2020 ACM/IEEE International Conference on Human-robot Interaction (Cambridge, United Kingdom) (HRI'20). Association for Computing Machinery, New York, NY, 289-297. https://doi.org/10.1145/3319502.3374795

[148] Laurel D. Riek. 2012. Wizard of oz studies in HRI: A systematic review and new reporting guidelines. F. Hum.-robot Interact. 1, 1 (July 2012), 119-136. https://doi.org/10.5898/JHRI.1.1.Riek

[149] Nina Riether, Frank Hegel, Britta Wrede, and Gernot Horstmann. 2012. Social facilitation with social robots? In Proceedings of the 7th Annual ACM/IEEE International Conference on Human-robot Interaction (Boston, Massachusetts) (HRI'12). ACM, New York, NY, 41-48. https://doi.org/10.1145/2157689.2157697

[150] David A. Robb, Muneeb I. Ahmad, Carlo Tiseo, Simona Aracri, Alistair C. McConnell, Vincent Page, Christian Dondrup, Francisco J. Chiyah Garcia, Hai-Nguyen Nguyen, Ėric Pairet, Paola Ardón Ramírez, Tushar Semwal, Hazel M. Taylor, Lindsay J. Wilson, David Lane, Helen Hastie, and Katrin Lohan. 2020. Robots in the danger zone: Exploring public perception through engagement. In Proceedings of the 2020 ACM/IEEE International Conference on Human-robot Interaction (Cambridge, United Kingdom) (HRI'20). Association for Computing Machinery, New York, NY, 93-102. https://doi.org/10.1145/3319502.3374789

[151] David Robert and Cynthia Breazeal. 2012. Blended reality characters. In Proceedings of the 7th Annual ACM/IEEE International Conference on Human-robot Interaction (Boston, Massachusetts) (HRI'12). ACM, New York, NY, 359366. https://doi.org/10.1145/2157689.2157810

[152] Pierre Rouanet, Fabien Danieau, and Pierre-Yves Oudeyer. 2011. A robotic game to evaluate interfaces used to show and teach visual objects to a robot in real world condition. In Proceedings of the 6th International Conference on Human-robot Interaction (Lausanne, Switzerland) (HRI'11). ACM, New York, NY, 313-320. https://doi.org/10.1145/ 1957656.1957782 
[153] Adam Rule and Jodi Forlizzi. 2012. Designing interfaces for multi-user, multi-robot systems. In Proceedings of the 7th Annual ACM/IEEE International Conference on Human-robot Interaction (Boston, Massachusetts) (HRI'12). ACM, New York, NY, 97-104. https://doi.org/10.1145/2157689.2157705

[154] Alessandra Maria Sabelli, Takayuki Kanda, and Norihiro Hagita. 2011. A conversational robot in an elderly care center: An ethnographic study. In Proceedings of the 6th International Conference on Human-robot Interaction (Lausanne, Switzerland) (HRI'11). ACM, New York, NY, 37-44. https://doi.org/10.1145/1957656.1957669

[155] Nicole Salomons, Michael van der Linden, Sarah Strohkorb Sebo, and Brian Scassellati. 2018. Humans conform to robots: Disambiguating trust, truth, and conformity. In Proceedings of the 2018 ACM/IEEE International Conference on Human-robot Interaction (Chicago, IL) (HRI'18). ACM, New York, NY, 187-195. https://doi.org/10.1145/3171221. 3171282

[156] Anara Sandygulova, Wafa Johal, Zhanel Zhexenova, Bolat Tleubayev, Aida Zhanatkyzy, Aizada Turarova, Zhansaule Telisheva, Anna CohenMiller, Thibault Asselborn, and Pierre Dillenbourg. 2020. Cowriting Kazakh: Learning a new script with a robot. In Proceedings of the 2020 ACM/IEEE International Conference on Human-robot Interaction (Cambridge, United Kingdom) (HRI'20). Association for Computing Machinery, New York, NY, 113-120. https: //doi.org/10.1145/3319502.3374813

[157] Stephanie Santosa and Daniel Wigdor. 2013. A field study of multi-device workflows in distributed workspaces. In Proceedings of the 2013 ACM International foint Conference on Pervasive and Ubiquitous Computing. ACM, New York, 63-72.

[158] Thorsten Schodde, Kirsten Bergmann, and Stefan Kopp. 2017. Adaptive robot language tutoring based on Bayesian knowledge tracing and predictive decision-making. In Proceedings of the 2017 ACM/IEEE International Conference on Human-robot Interaction (Vienna, Austria) (HRI'17). ACM, New York, NY, 128-136. https://doi.org/10.1145/2909824. 3020222

[159] Google Scholar. 2020. Top publications: Human-computer interaction. https://scholar.google.com/citations?view_ op=top_venues\&hl=en\&vq=eng_humancomputerinteraction.

[160] Trenton Schulz, Jim Torresen, and Jo Herstad. 2019. Animation techniques in human-robot interaction user studies: A systematic literature review. f. Hum.-robot Interact. 8, 2, Article 12 (June 2019), 22 pages. https://doi.org/10.1145/ 3317325

[161] Sarah Sebo, Brett Stoll, Brian Scassellati, and Malte F. Jung. 2020. Robots in groups and teams: A literature review. In Proceedings of the ACM Human-computer Interactaton 4, CSCW2, Article 176 (Oct. 2020), 36 pages. https://doi.org/ $10.1145 / 3415247$

[162] S. S. Sebo, P. Krishnamurthi, and B. Scassellati. 2019. "I don't believe you”: Investigating the effects of robot trust violation and repair. In Proceedings of the 2019 14th ACM/IEEE International Conference on Human-robot Interaction (HRI'19). ACM, New York, 57-65. https://doi.org/10.1109/HRI.2019.8673169

[163] Brennan P. Sellner, Laura M. Hiatt, Reid Simmons, and Sanjiv Singh. 2006. Attaining situational awareness for sliding autonomy. In Proceedings of the 1st ACM SIGCHI/SIGART Conference on Human-robot Interaction (Salt Lake City, Utah, USA) (HRI'06). ACM, New York, NY, 80-87. https://doi.org/10.1145/1121241.1121257

[164] Pedro Sequeira, Patrícia Alves-Oliveira, Tiago Ribeiro, Eugenio Di Tullio, Sofia Petisca, Francisco S. Melo, Ginevra Castellano, and Ana Paiva. 2016. Discovering social interaction strategies for robots from restricted-perception Wizard-of-Oz studies. In Proceedings of the 11th ACM/IEEE International Conference on Human Robot Interaction (Christchurch, New Zealand) (HRI'16). IEEE Press, Piscataway, NJ, 197-204. http://dl.acm.org/citation.cfm?id= 2906831.2906866.

[165] Solace Shen, Petr Slovak, and Malte F. Jung. 2018. "Stop. I see a conflict Happening”: A robot mediator for young children's interpersonal conflict resolution. In Proceedings of the 2018 ACM/IEEE International Conference on Humanrobot Interaction (Chicago, IL) (HRI'18). ACM, New York, NY, 69-77. https://doi.org/10.1145/3171221.3171248

[166] Masahiro Shiomi, Takayuki Kanda, Hiroshi Ishiguro, and Norihiro Hagita. 2006. Interactive humanoid robots for a science museum. In Proceedings of the 1st ACM SIGCHI/SIGART Conference on Human-robot Interaction (Salt Lake City, Utah) (HRI'06). ACM, New York, NY, 305-312. https://doi.org/10.1145/1121241.1121293

[167] Masahiro Shiomi, Takayuki Kanda, Hiroshi Ishiguro, and Norihiro Hagita. 2010. A larger audience, please!: Encouraging people to listen to a guide robot. In Proceedings of the 5th ACM/IEEE International Conference on Human-robot Interaction (Osaka, Japan) (HRI'10). IEEE Press, Piscataway, NJ, 31-38. http://dl.acm.org/citation.cfm?id=1734454.1734469.

[168] Masahiro Shiomi, Takayuki Kanda, Satoshi Koizumi, Hiroshi Ishiguro, and Norihiro Hagita. 2007. Group attention control for communication robots with Wizard of $\mathrm{Oz}$ approach. In Proceedings of the ACM/IEEE International Conference on Human-robot Interaction (Arlington, Virginia) (HRI'07). ACM, New York, NY, 121-128. https: //doi.org/10.1145/1228716.1228733

[169] Masahiro Shiomi, Daisuke Sakamoto, Takayuki Kanda, Carlos Toshinori Ishi, Hiroshi Ishiguro, and Norihiro Hagita. 2008. A semi-autonomous communication robot: A field trial at a train station. In Proceedings of the 3rd ACM/IEEE International Conference on Human Robot Interaction (Amsterdam, The Netherlands) (HRI'08). ACM, New York, NY, 303-310. https://doi.org/10.1145/1349822.1349862 
[170] David Sirkin and Wendy Ju. 2012. Consistency in physical and on-screen action improves perceptions of tele- presence robots. In Proceedings of the 7th Annual ACM/IEEE International Conference on Human-robot Interaction (Boston, Massachusetts) (HRI'12). ACM, New York, NY, 57-64. https://doi.org/10.1145/2157689.2157699

[171] Gabriel Skantze. 2017. Predicting and regulating participation equality in human-robot conversations: Effects of age and gender. In Proceedings of the 2017 ACM/IEEE International Conference on Human-robot Interaction (Vienna, Austria) (HRI'17). ACM, New York, NY, 196-204. https://doi.org/10.1145/2909824.3020210

[172] Henrik Sørensen, Dimitrios Raptis, Jesper Kjeldskov, and Mikael B. Skov. 2014. The 4C framework: Principles of interaction in digital ecosystems. In Proceedings of the 2014 ACM International foint Conference on Pervasive and Ubiquitous Computing (Seattle, Washington) (UbiComp'14). ACM, New York, NY, 87-97. https://doi.org/10.1145/2632048.2636089

[173] Maj Stenmark, Mathias Haage, and Elin Anna Topp. 2017. Simplified programming of re-usable skills on a safe industrial robot: Prototype and evaluation. In Proceedings of the 2017 ACM/IEEE International Conference on Humanrobot Interaction (Vienna, Austria) (HRI'17). ACM, New York, NY, 463-472. https://doi.org/10.1145/2909824.3020227

[174] Brett Stoll, Samantha Reig, Lucy He, Ian Kaplan, Malte F. Jung, and Susan R. Fussell. 2018. Wait, can you move the robot? Examining telepresence robot use in collaborative teams. In Proceedings of the 2018 ACM/IEEE International Conference on Human-robot Interaction (Chicago, IL) (HRI'18). ACM, New York, NY, 14-22. https://doi.org/10.1145/ 3171221.3171243

[175] Megan Strait, Cody Canning, and Matthias Scheutz. 2014. Let me tell you! Investigating the effects of robot communication strategies in advice-giving situations based on robot appearance, interaction modality and distance. In Proceedings of the 2014 ACM/IEEE International Conference on Human-robot Interaction (Bielefeld, Germany) (HRI'14). ACM, New York, NY, 479-486. https://doi.org/10.1145/2559636.2559670

[176] Sarah Strohkorb Sebo, Ling Liang Dong, Nicholas Chang, and Brian Scassellati. 2020. Strategies for the inclusion of human members within human-robot teams. In Proceedings of the 2020 ACM/IEEE International Conference on Human-robot Interaction (Cambridge, United Kingdom) (HRI'20). Association for Computing Machinery, New York, NY, 309-317. https://doi.org/10.1145/3319502.3374808

[177] Sarah Strohkorb Sebo, Margaret Traeger, Malte Jung, and Brian Scassellati. 2018. The ripple effects of vulnerability: The effects of a robot's vulnerable behavior on trust in human-robot teams. In Proceedings of the 2018 ACM/IEEE International Conference on Human-robot Interaction (Chicago, IL, USA) (HRI'18). ACM, New York, NY, USA, 178-186. https://doi.org/10.1145/3171221.3171275

[178] Leila Takayama and Helen Harris. 2013. Presentation of (Telepresent) Self: On the Double-edged Effects of Mirrors. In Proceedings of the 8th ACM/IEEE International Conference on Human-robot Interaction (Tokyo, Japan) (HRI'13). IEEE Press, Piscataway, NJ, USA, 381-388. http://dl.acm.org/citation.cfm?id=2447556.2447684.

[179] X. Z. Tan, S. Reig, E. J. Carter, and A. Steinfeld. 2019. From one to another: How robot-robot interaction affects users' perceptions following a transition between robots. In Proceedings of the 2019 14th ACM/IEEE International Conference on Human-robot Interaction (HRI'19). 114-122. https://doi.org/10.1109/HRI.2019.8673304

[180] Xiang Zhi Tan, Marynel Vázquez, Elizabeth J. Carter, Cecilia G. Morales, and Aaron Steinfeld. 2018. Inducing bystander interventions during robot abuse with social mechanisms. In Proceedings of the 2018 ACM/IEEE International Conference on Human-robot Interaction (Chicago, IL) (HRI'18). ACM, New York, NY, 169-177. https://doi.org/10.1145/ 3171221.3171247

[181] Fumihide Tanaka, Javier R. Movellan, Bret Fortenberry, and Kazuki Aisaka. 2006. Daily HRI evaluation at a classroom environment: Reports from dance interaction experiments. In Proceedings of the 1st ACM SIGCHI/SIGART Conference on Human-robot Interaction (Salt Lake City, Utah) (HRI'06). ACM, New York, NY, 3-9. https://doi.org/10.1145/1121241. 1121245

[182] Fumihide Tanaka, Toshimitsu Takahashi, Shizuko Matsuzoe, Nao Tazawa, and Masahiko Morita. 2014. Telepresence robot helps children in communicating with teachers who speak a different language. In Proceedings of the 2014 ACM/IEEE International Conference on Human-robot Interaction (Bielefeld, Germany) (HRI'14). ACM, New York, NY, 399-406. https://doi.org/10.1145/2559636.2559654

[183] Kazuaki Tanaka, Naomi Yamashita, Hideyuki Nakanishi, and Hiroshi Ishiguro. 2016. Teleoperated or autonomous? How to produce a robot operator's pseudo presence in HRI. In Proceedings of the 11th ACM/IEEE International Conference on Human-robot Interaction (Christchurch, New Zealand) (HRI'16). IEEE Press, Piscataway, NJ, 133-140. http://dl.acm.org/citation.cfm?id=2906831.2906856.

[184] H. Tennent, S. Shen, and M. Jung. 2019. Micbot: A peripheral robotic object to shape conversational dynamics and team performance. In Proceedings of the 2019 14th ACM/IEEE International Conference on Human-robot Interaction (HRI'19). ACM, New York, NY, 133-142. https://doi.org/10.1109/HRI.2019.8673013

[185] Lucia Terrenghi, Aaron Quigley, and Alan Dix. 2009. A taxonomy for and analysis of multi-person-display ecosystems. Personal and Ubiquitous Computing 13, 8 (2009), 583.

[186] R. Thiessen, D. J. Rea, D. S. Garcha, C. Cheng, and J. E. Young. 2019. Infrasound for HRI: A robot using low-frequency vibrations to impact how people perceive its actions. In Proceedings of the 2019 14th ACM/IEEE International Conference on Human-robot Interaction (HRI). ACM, New York,11-18. https://doi.org/10.1109/HRI.2019.8673172 
[187] Myrthe Tielman, Mark Neerincx, John-Jules Meyer, and Rosemarijn Looije. 2014. Adaptive emotional expression in robot-child interaction. In Proceedings of the 2014 ACM/IEEE International Conference on Human-robot Interaction (Bielefeld, Germany) (HRI'14). ACM, New York, NY, 407-414. https://doi.org/10.1145/2559636.2559663

[188] Katherine M. Tsui, Munjal Desai, Holly A. Yanco, and Chris Uhlik. 2011. Exploring use cases for telepresence robots. In Proceedings of the 6th International Conference on Human-robot Interaction (Lausanne, Switzerland) (HRI'11). ACM, New York, NY, 11-18. https://doi.org/10.1145/1957656.1957664

[189] D. Utami and T. Bickmore. 2019. Collaborative user responses in multiparty interaction with a couples counselor robot. In Proceedings of the 2019 14th ACM/IEEE International Conference on Human-robot Interaction (HRI'19). ACM, New York, NY, 294-303. https://doi.org/10.1109/HRI.2019.8673177

[190] Aris Valtazanos and Subramanian Ramamoorthy. 2013. Evaluating the effects of limited perception on interactive decisions in mixed robotic domains. In Proceedings of the 8th ACM/IEEE International Conference on Human-robot Interaction (Tokyo, Japan) (HRI'13). IEEE Press, Piscataway, NJ, 9-16. http://dl.acm.org/citation.cfm?id=2447556.2447559.

[191] Esther J. G. van der Drift, Robbert-Jan Beun, Rosemarijn Looije, Olivier A. Blanson Henkemans, and Mark A. Neerincx. 2014. A remote social robot to motivate and support diabetic children in keeping a diary. In Proceedings of the 2014 ACM/IEEE International Conference on Human-robot Interaction (Bielefeld, Germany) (HRI'14). ACM, New York, NY, 463-470. https://doi.org/10.1145/2559636.2559664

[192] Peggy van Minkelen, Carmen Gruson, Pleun van Hees, Mirle Willems, Jan de Wit, Rian Aarts, Jaap Denissen, and Paul Vogt. 2020. Using self-determination theory in social robots to increase motivation in L2 word learning. In Proceedings of the 2020 ACM/IEEE International Conference on Human-robot Interaction (Cambridge, United Kingdom) (HRI'20). Association for Computing Machinery, New York, NY, 369-377. https://doi.org/10.1145/3319502.3374828

[193] Marynel Vázquez, Elizabeth J. Carter, Braden McDorman, Jodi Forlizzi, Aaron Steinfeld, and Scott E. Hudson. 2017. Towards robot autonomy in group conversations: Understanding the effects of body orientation and gaze. In Proceedings of the 2017 ACM/IEEE International Conference on Human-robot Interaction (Vienna, Austria) (HRI'17). ACM, New York, NY, 42-52. https://doi.org/10.1145/2909824.3020207

[194] Marynel Vázquez, Aaron Steinfeld, Scott E. Hudson, and Jodi Forlizzi. 2014. Spatial and other social engagement cues in a child-robot interaction: Effects of a sidekick. In Proceedings of the 2014 ACM/IEEE International Conference on Human-robot Interaction (Bielefeld, Germany) (HRI'14). ACM, New York, NY, 391-398. https://doi.org/10.1145/ 2559636.2559684

[195] John Vilk and Naomi T. Fitter. 2020. Comedians in cafes getting data: Evaluating timing and adaptivity in realworld robot comedy performance. In Proceedings of the 2020 ACM/IEEE International Conference on Human-robot Interaction (Cambridge, United Kingdom) (HRI'20). Association for Computing Machinery, New York, NY, 223-231. https://doi.org/10.1145/3319502.3374780

[196] P. Vogt, R. van den Berghe, M. de Haas, L. Hoffman, J. Kanero, E. Mamus, J. Montanier, C. Oranç, O. Oudgenoeg-Paz, D. H. García, F. Papadopoulos, T. Schodde, J. Verhagen, C. D. Wallbridgell, B. Willemsen, J. de Wit, T. Belpaeme, T. Göksun, S. Kopp, E. Krahmer, A. C. Küntay, P. Leseman, and A. K. Pandey. 2019. Second language tutoring using social robots: A large-scale study. In Proceedings of the 2019 14th ACM/IEEE International Conference on Human-robot Interaction (HRI'19). ACM, New York, NY, 497-505. https://doi.org/10.1109/HRI.2019.8673077

[197] Michael Walker, Hooman Hedayati, Jennifer Lee, and Daniel Szafir. 2018. Communicating robot motion intent with augmented reality. In Proceedings of the 2018 ACM/IEEE International Conference on Human-robot Interaction (Chicago, IL) (HRI'18). ACM, New York, NY, 316-324. https://doi.org/10.1145/3171221.3171253

[198] M. E. Walker, H. Hedayati, and D. Szafir. 2019. Robot teleoperation with augmented reality virtual surrogates. In Proceedings of the 2019 14th ACM/IEEE International Conference on Human-robot Interaction (HRI'19). ACM, New York, NY, 202-210. https://doi.org/10.1109/HRI.2019.8673306

[199] Jijun Wang and Michael Lewis. 2008. Assessing cooperation in human control of heterogeneous robots. In Proceedings of the 3rd ACM/IEEE International Conference on Human Robot Interaction (Amsterdam, The Netherlands) (HRI'08). ACM, New York, NY, 9-16. https://doi.org/10.1145/1349822.1349825

[200] Lin Wang, Pei-Luen Patrick Rau, Vanessa Evers, Benjamin Krisper Robinson, and Pamela Hinds. 2010. When in Rome: The Role of Culture: Context in Adherence to Robot Recommendations. In Proceedings of the 5th ACM/IEEE International Conference on Human-robot Interaction (Osaka, Japan) (HRI'10). IEEE Press, Piscataway, NJ, 359-366. http://dl.acm.org/citation.cfm?id=1734454.1734578.

[201] Sachie Yamada, Takayuki Kanda, and Kanako Tomita. 2020. An escalating model of children's robot abuse. In Proceedings of the 2020 ACM/IEEE International Conference on Human-robot Interaction (Cambridge, United Kingdom) (HRI'20). Association for Computing Machinery, New York, NY, 191-199. https://doi.org/10.1145/3319502.3374833

[202] Fumitaka Yamaoka, Takayuki Kanda, Hiroshi Ishiguro, and Norihiro Hagita. 2009. Developing a model of robot behavior to identify and appropriately respond to implicit attention-shifting. In Proceedings of the 4th ACM/IEEE International Conference on Human Robot Interaction (La Jolla, California) (HRI'09). ACM, New York, NY, 133-140. https://doi.org/10.1145/1514095.1514120 
[203] Akiko Yamazaki, Keiichi Yamazaki, Takaya Ohyama, Yoshinori Kobayashi, and Yoshinori Kuno. 2012. A technosociological solution for designing a museum guide robot: Regarding choosing an appropriate visitor. In Proceedings of the 7th Annual ACM/IEEE International Conference on Human-robot Interaction (Boston, Massachusetts) (HRI'12). ACM, New York, NY, 309-316. https://doi.org/10.1145/2157689.2157800

[204] Holly A. Yanco and Jill Drury. 2004. Classifying human-robot interaction: An updated taxonomy. In Proceedings of the 2004 IEEE International Conference on Systems, Man and Cybernetics (IEEE Cat. No. 04CH37583), Vol. 3. IEEE, 2841-2846.

[205] Debora Zanatto, Massimiliano Patacchiola, Jeremy Goslin, Serge Thill, and Angelo Cangelosi. 2020. Do humans imitate robots? An investigation of strategic social learning in human-robot interaction. In Proceedings of the 2020 ACM/IEEE International Conference on Human-robot Interaction (Cambridge, United Kingdom) (HRI'20). Association for Computing Machinery, New York, NY, 449-457. https://doi.org/10.1145/3319502.3374776

[206] Kuanhao Zheng, Dylan F. Glas, Takayuki Kanda, Hiroshi Ishiguro, and Norihiro Hagita. 2011. How many social robots can one operator control? In Proceedings of the 6th International Conference on Human-robot Interaction (Lausanne, Switzerland) (HRI'11). ACM, New York, NY, 379-386. https://doi.org/10.1145/1957656.1957794

Received December 2020; accepted August 2021 